\title{
A scoping review of risk behaviour interventions in young men
}

Lee M Ashton', Melinda J Hutchesson', Megan E Rollo', Philip J Morgan² and Clare E Collins ${ }^{1 *}$

\begin{abstract}
Background: Young adult males commonly engage in risky behaviours placing them at risk of acute and chronic health conditions. The purpose of this scoping review was to provide an overview of existing literature, describing the interventions targeting risk behaviours in young adult males.
\end{abstract}

Methods: A search of seven electronic databases, grey literature and relevant journals reported in English language until May 2013 was conducted. All interventions that promoted healthy behaviours or reductions in risky behaviours to treat or prevent an associated health issue(s) in young adult males (17-35 years) in upper-middle and high-income countries were included. For inclusion the appropriate age range had to be reported and the sample had to be young adult male participants only or the outcomes reported with stratification by age and/or sex to include young adult males. Risk behaviours included: physical inactivity, poor diet, alcohol use, tobacco smoking, recreational drug use, unsafe sexual behaviours, tanning/sun exposure, violence, unsafe vehicle driving, gambling and self-harm.

Results: The search strategy identified 16,739 unique citations and the full-text of 1149 studies were retrieved and screened with 100 included studies focussed on: physical inactivity (27\%), alcohol use (25\%), unsafe sexual behaviour (21\%), poor diet (5\%), unsafe vehicle driving (5\%), tobacco smoking (4\%), recreational drug use (2\%), and tanning/sun exposure (1\%) with no relevant studies targeting violence, gambling or self-harm. Also 10\% of the studies targeted multiple risk behaviours. The most common study design was randomized controlled trials (62\%). Face-to-face was the most common form of intervention delivery (71\%) and the majority were conducted in university/college settings (46\%). There were 46 studies (46\%) that included young adult male participants only, the remaining studies reported outcomes stratified by age and/or sex.

Conclusion: Risk behaviours in young men have been targeted to some extent, but the amount of research varies across risk behaviours. There is a need for more targeted and tailored interventions that seek to promote healthy behaviours or decrease risky behaviours in young men.

Keywords: Scoping review, Young men, Risk behaviours, Health behaviour, Risk reduction behaviour, Intervention

\section{Background}

Risk behaviours are defined as actions that potentially have adverse effects on health and increase the likelihood of developing a specific disease [1]. Examples include: physical inactivity, unhealthy dietary habits, unsafe sexual behaviours, tobacco smoking, alcohol use, recreational drug use, unsafe vehicle driving, violence [2], self-harm [3], gambling [4] and tanning/sun exposure [5]. Risk behaviours contribute immensely to morbidity and mortality

\footnotetext{
* Correspondence: clare.collins@newcastle.edu.au

${ }^{1}$ School of Health Sciences, Faculty of Health and Medicine, Priority Research Centre for Physical Activity and Nutrition, University of Newcastle, Callaghan Campus, Newcastle, Australia

Full list of author information is available at the end of the article
}

in men worldwide with tobacco use, physical inactivity and low fruit and vegetable intake responsible for $11 \%, 5 \%$ and $3 \%$ of total mortality globally, respectively [6]. The leading risks contributing to the global burden of disease in males include alcohol use, tobacco smoking and unsafe sex with each contributing $7 \%, 5 \%$ and $4 \%$ to total Disability-Adjusted Life Years (DALYs) [6]. Despite this, interventions targeting specific risk behaviours have not been consistently efficacious in achieving positive health behaviour change [7]. For instance, a meta-synthesis of 62 meta-analyses established that interventions targeting several behaviours (diet, physical activity, sexual behaviours and addictive behaviours) at individual-level, group-level 
and population-level had small to medium effect sizes (range $=0.08-0.45$, mean effect size $=0.21$ ) with the efficacy varying across the different behaviour domains [7].

A key concern is the young age at which individuals commence risky behaviours. Recent estimates indicate that one-third of the total disease burden in adults is due to risk behaviours that start in youth [2]. Engaging in risk behaviours at an early age is related to detrimental impacts on health including obesity, sexually transmitted diseases and specific cancers [8-10]. Research in the USA has shown that mortality from risk behaviours (suicide, motor vehicle accidents and unintentional injuries) in young adult males is 137 per 100,000 deaths, almost three times greater than for young females [11]. In addition the prevalence of obesity in young men is a concern; in the USA 29\% (aged 20-39 yr) are classed as obese [12]. In Australia 15\% (aged 18-24 yr) and 23\% (aged 25-34 yr) are obese [13] and in England 12\% (aged 16-24 yr) and 17\% (aged 25-34 yr) are obese [14]. Moreover, contributors to obesity include physical inactivity, with approximately 20\% of young men (aged 18-39 yr) failing to meet the national targets [15]. Poor diet quality, characterised by high intakes of energy dense foods and inadequate intakes of key food groups such as fruit, vegetables, wholegrain, legumes and nuts also contribute to the high prevalence of obesity [10]. In the USA $89 \%$ of young men (aged 19-30 yr) are failing to consume minimum recommended intakes for total fruit and 95\% for total vegetables [16]. Similar inadequacies are apparent in Australia with $97 \%$ of young men (18-24 yr) failing to meet national recommendations for fruit and vegetables [13].

Tobacco smoking is the leading risk factor for global mortality in men [6]; $22 \%$ of young Australian men (aged 18-24 yr) [13] and 20\% of young men from the USA [17] are classed as current daily tobacco smokers. Alcohol misuse is the third leading risk factor for global mortality in males (6.2\% of deaths) [6]. Half (52.3\%) of USA young adult men exceed daily maximum recommended alcohol intakes [18]. Alcohol consumption may be associated with other risky behaviours as it reduces inhibitions thereby increasing the likelihood of other behaviours including tobacco smoking, injuries, driving under the influence, violence and unsafe sex $[2,9,19,20]$.

Gambling behaviours are also a concern in young adults with almost $10 \%$ of college students in the USA and Canada qualifying as problem gamblers and $6 \%$ as pathological gamblers [4]. Sun exposure is another risk behaviour of concern in USA young men as they account for more than $60 \%$ of melanoma deaths [5] which is the most common form of cancer in young adults [21].

In young adult men social influences such as peer pressure [22], and environmental changes including leaving home, starting tertiary education, cohabitation and beginning employment [23] may provide some explanation for the widespread prevalence of risk behaviours in this population. Early work linking masculinity with men's health often associated dominant forms of masculinity (i.e. Hegemonic masculinity) with poorer health behaviours [24-26] Despite this, hegemonic masculinity is often encouraged in society amongst young men e.g. "take it like a man" [27] and in young men physical risk is often promoted and celebrated [28]. Young men who align to hegemonic masculinity may feel they are 'invincible' and hence take risks without fear of consequences [29], which supports the notion that hegemonic masculinities are strong predictors of risk behaviours [30]. This is not to say this is the case for all young men but may provide justification for the undertaking of risk behaviours in particular subgroups of young men.

We need greater understanding of how sex and gender influences risky behaviours and health practices. Health research often fails to consider sex and gender in design, data collection and/or analysis; instead a generic 'one size fits all' approach is often adopted [31]. There is a need for more targeted interventions (i.e. those intended to reach a specific population subgroup [32]) and tailored interventions (those intended to reach one specific person, based on known differences that exist between individuals [32]) in young men because men's needs and behaviours differ across the lifespan [33] and are different to women [34]. By utilizing a targeted and/or tailored approach the unique needs and interests of young men can be accounted for and help achieve the desired behaviour change [35]. According to the 'elaboration likelihood model' [36] more people are likely to process and retain information if they perceive it to be personally relevant, thus increasing the likelihood of attitude change [37]. In interventions directed at overweight/ obesity [38], physical activity [39] and smoking cessation [40] research has confirmed the need for targeted and/or tailored approaches to engage men. Research has also drawn attention to the evidence gap for young men by emphasizing the need for targeted and/or tailored interventions in obesity treatment [41] and prevention [42], mental health [43] unsafe sexual behaviour and HIV prevention [44] and alcohol use [45]. Therefore, the purpose of this scoping review is to provide a broad overview of interventions that have either examined risk behaviours exclusively in young adult male population groups and those that have reported outcomes stratified by age and/ or sex to identify outcomes in young adult males. The scoping review design will allow examination of the extent, range and nature of research activity, will help to summarise and disseminate research findings and can also serve as a foundation for more detailed systematic reviews [46]. Any identified gaps in the literature can guide future research of risk behaviour interventions in young men. 


\section{Methods}

The methodological framework as proposed by Arksey and O'Malley [46] was used to guide the scoping review. This framework provides a robust foundation for scoping study methodology and guidance to researchers undertaking scoping reviews [46]. The components of the framework used to guide this scoping review include: identifying the research question, searching for relevant studies, selecting studies, charting the data and collating, summarizing, and reporting the results.

\section{Identification of the research question to be addressed}

The research question was defined using the PICOS (Population-Intervention-Comparison-Outcome-Study design) format. The question was designed to be broad and comprehensive to capture the full breadth of the literature and is a key aspect in scoping the field [46].

\section{Participants}

A population of young adult males (aged 17-35 years) was required for inclusion, with the appropriate age range specified within the article. Both the National Institute of Health [47] and the European commission of Men's health [29] have used 18-35 years to define young adults. The inclusion criteria for participants was shaped around this definition, but the rationale for including age 17 was due to unsafe vehicle driving, as this is the age a driving license can be obtained in most countries. Studies were excluded if all participants had a major chronic medical condition (s). Research from lower income countries and low-tomiddle income countries were excluded because of the large heterogeneity between low-income/low-to-middle income and upper-middle/high-income countries (e.g. the intervention characteristics of a HIV prevention intervention in a low-income/low-to-middle income country is unlikely to be translatable to an upper-middle/high-income country). The income group of each country was determined from the World Bank Group website [48]. Studies containing both males and females were included if results had been stratified by young adult males. Similarly studies containing old and young adults were only included if results had been stratified by young adult males. Stratified results specifically for young adult males must be reported in the Results section, as opposed to including 'sex' in regression models.

\section{Interventions}

Interventions designed to promote healthy behaviour (specific to the behaviours outlined in Table 1) or decrease risky behaviour to treat or prevent the associated health problem(s) (Table 1) in young adult males were accepted for inclusion. Any interventions targeting the risk behaviours as outlined in the World Health Organization were accepted for inclusion [2]. Gambling, tanning/sun exposure

Table 1 Included outcomes of interest

\begin{tabular}{ll}
\hline Risk behaviour & Associated health outcomes \\
\hline Physical inactivity & $\begin{array}{l}\text { Obesity, diabetes, hypertension, } \\
\text { hyperglycaemia, cancer, CVD, mental } \\
\text { health disorders (i.e. depression), } \\
\text { quality of life. }\end{array}$
\end{tabular}

Poor diet

Obesity, diabetes, hypercholesterolemia, hypertension, hyperglycaemia, CVD, quality of life.

Alcohol use

Actual injury, alcohol dependence, obesity, diabetes, mental health disorders (i.e. depression), cancer, liver disease, CVD, quality of life.

Tobacco smoking

Respiratory/lung diseases, cancer, CVD, hypertension, hyperglycaemia, quality of life.

Recreational drug use

Sexual behaviours

Violence

Unsafe vehicle driving

Gambling

Tanning/ sun exposure

Self-harm
Mental health disorders (i.e. depression, anxiety), HIV, hepatitis.

HIV, STD's.

Actual injury, mental health disorders (i.e ADHD, schizophrenia, depression etc)

Actual injury, mental health disorders (i.e ADHD, bi-polar, schizophrenia, anxiety etc)

Mental health disorders (i.e. depression), quality of life.

Skin cancer

Mental health disorders (i.e. depression), quality of life.

and self-harm were also included due to their high prevalence rates in young men $[4,5,11,21,49]$. In addition, interventions targeting multiple risk behaviours were included.

\section{Outcomes of interest}

To be included, the intervention had to aim to change one of the risk behaviours and have measured as an outcome change in that risk behaviour and/or the associated health outcome (Table 1).

\section{Study design}

The following experimental study designs were included in the review: randomized controlled trials, cluster randomized controlled trials, cluster controlled studies, nonrandomized controlled trials (including quasi-experimental or controlled clinical trial), case series (including pre-test/ post-test design or before-after studies with no control) and interrupted time series. Systematic reviews and meta- 
analyses were included in the search strategy so that the reference lists of relevant reviews could be searched for additional relevant articles.

\section{Identification of studies relevant to the research question Search strategy}

The databases searched were: MEDLINE (Ovid) MEDLINE in process (Ovid), EMBASE (Ovid), PsycINFO (Ovid), Science Citation Index (WoS), Cinahl (EbscoHost) and Cochrane Library (Wiley). To locate relevant grey literature we used the 'Grey Matters search tool,' 'The grey literature report,' 'Open Grey' and the clinical trials registry (clinicaltrials.gov). All sources were searched from the date of inception to May 2013. Restrictions were applied to include only English language articles and human participants. The search strategy was developed by the research team with consultation from a medical librarian. The search consisted of focussed 'text word' searches and subject heading searches $(\mathrm{MeSH})$. Our search strategy from the electronic databases can be found in Additional file 1. In addition all of the conference proceeding abstracts picked up in the bibliographic databases were assessed for inclusion and a reference list search of included studies, relevant systematic reviews and meta-analyses were conducted. We also searched the following key male health journals: International Journal of Men's Health, Journal of Men's Health and American Journal of Men's Health from the first published article to the most recent published articles (Earliest: February 2004 and latest: November 2013).

\section{Selection of studies to include in the review}

After removal of duplicates one reviewer conducted a screening review of the titles and excluded those which did not meet the inclusion criteria. One reviewer screened the abstracts and divided into three categories (Category 1: Relevant, Category 2: Not relevant, Category 3: Potentially relevant but failed to specify if results were stratified by age and/or sex). For those in category 3 we randomly selected and retrieved $10 \%(n=104)$ of the full text articles to identify if results have been stratified by age and/or sex. None of these articles were deemed relevant after full text screening so the remaining articles in category 3 were excluded without further review. A second reviewer independently reviewed $10 \%$ of all abstracts $(n=1232)$ and an agreement of $97.6 \%$ was achieved. A third reviewer was consulted to resolve any disagreements. The full text articles of studies that were believed to satisfy the inclusion criteria (Category 1) were retrieved and a final decision on inclusion was made by one reviewer with a second reviewer consulted for any uncertainties. A third reviewer independently reviewed $10 \%$ of all full text papers $(n=115)$ and an agreement of $100 \%$ was achieved.

\section{Charting of information and data within the included studies}

Data were extracted from included studies by one reviewer. For each study this included: author, year of publication, study design, risk behaviour targeted, associated health outcome targeted, target population group (e.g. young adult male participants only or results reported by strata for young adult males), sex and profession of intervention facilitator, sample age range, sample size, sample recruitment setting (e.g. university/college, community, hospital etc.), country of implementation, number of study arms, intervention strategy/study aim, theoretical model used to develop intervention, mode/delivery of intervention and intervention length and follow-up.

\section{Collating, summarising and reporting results of the review}

As is convention in scoping reviews, a numerical analysis was undertaken to elucidate the extent and nature of the studies [50].

\section{Results \\ Citation retrieval}

The search strategy identified 24,415 citations (Figure 1). After removal of duplicates 16,739 citations were screened based on title and subsequently the abstracts of 12,329 citations were examined. Following abstract review 1149 remained for full text screening, of which 1049 articles were excluded. This gave 100 studies included in the scoping review.

The 100 included studies appeared in 70 journals and the year of publication ranged from 1972-2013, with 72 studies $(72 \%)$ published from 2003 onwards. In addition one study was a grey literature paper, identified from the clinical trials registry (clinicaltrials.gov) [51]. The number of studies targeting each risk behaviour included 27 (27\%) for physical inactivity [52-78], 25 (25\%) for alcohol use [65-89], 21 (21\%) for unsafe sexual behaviour [51,79-98], 5 (5\%) for poor diet [99-103], 5 (5\%) for unsafe vehicle driving [104-108], 4 (4\%) for tobacco smoking [109-112], 2 (2\%) for recreational drug use [113,114] and 1 (1\%) for tanning/sun exposure [115]. There were 10 studies (10\%) that assessed multiple risk behaviours [116-125] (of these 3 studies targeted poor diet and physical inactivity $[120,122,123], 2$ studies targeted alcohol use and recreational drug use [117,121], 1 study targeted alcohol use and unsafe sexual behaviour [118], 1 study targeted poor diet, physical inactivity and tobacco smoking [116], 1 study targeted tobacco smoking, alcohol use and recreational drug use [119], 1 study targeted alcohol use and physical inactivity [125] and 1 study targeted unsafe vehicle driving and alcohol use [124]). No studies targeted the prevention or treatment of violence, gambling or self-harm. 


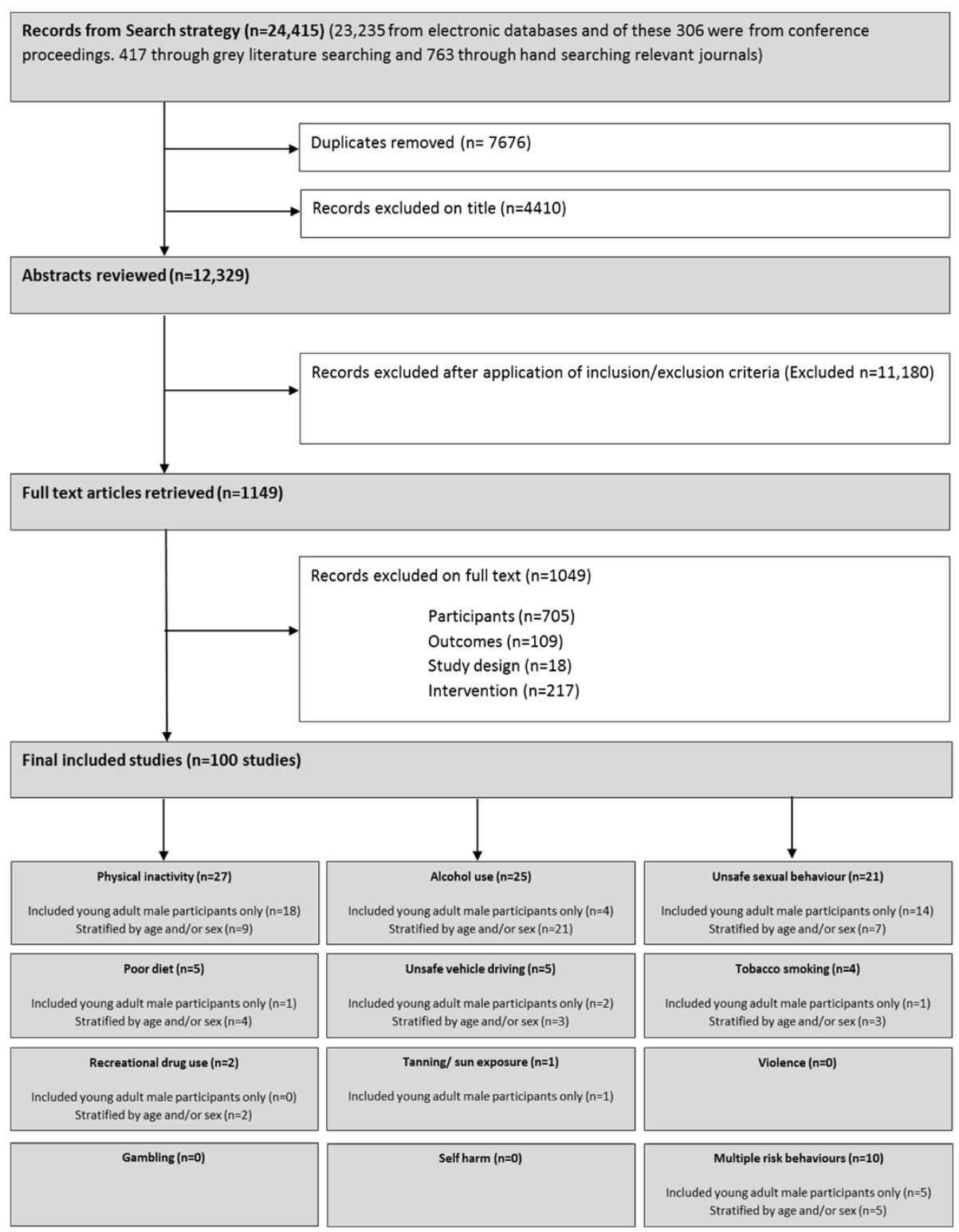

Figure 1 Flow chart of studies through the scoping review process.

Of the 100 included studies, 46 included young male participants only with 18 ( $18 \%$ of total studies and $39.1 \%$ of young adult male only studies) [52-54,57,58,60,61,64-73,76] for physical inactivity, 14 (14\% of total studies and $30.4 \%$ of young adult male only studies) for unsafe sexual behaviour [51,80,82,85-91,94,96-98], 4 (4\% of total studies and $8.7 \%$ of young adult male only studies) for alcohol use [126-129], 2 ( $2 \%$ of total studies and $4.3 \%$ of young adult male only studies) for unsafe vehicle driving $[104,105] 1$ (1\% of total studies and $2.2 \%$ of young adult male only studies) for tobacco smoking [110], 1 (1\% of total studies and $2.2 \%$ of young adult male only studies) for poor diet [103], and 1 (1\% of total studies and $2.2 \%$ of young adult male only studies) for tanning/sun exposure [115]. Also 5 (5\% of total studies and
$10.9 \%$ of young adult male only studies) of the studies with only young adult male participants targeted multiple risk behaviours [116,119,122,124,125]. See Additional file 2 for detailed information on the included studies, such as: author(s), title, year, study design, country, target population group, age range and risk behaviour targeted.

\section{Intervention country and risk behaviours}

The interventions were conducted across several continents, with most conducted in North America $(\mathrm{n}=63$, 63\%) $[45,51,57-59,62,63,68,73-75,77,78,80,82,85-94,96$, 97,99-101,103,106,109,111-113,115,117,118,121-123,125, 126,129-147] (the majority of these were conducted in the USA $n=61$ ). The remaining interventions were 
carried out in Europe $(\mathrm{n}=18,18 \%)[52,53,55,56,60,61$, $70,81,95,98,102,104,110,116,119,127,128,148]$, Australasia $(\mathrm{n}=8,8 \%) \quad[54,65,66,105,108,120,124,149]$, Asia $(\mathrm{n}=6$, $6 \%$ ) $[64,67,71,72,107,114]$, South America $(n=3,3 \%)$ $[69,79,83]$, Africa $(n=1,1 \%)[76]$ and one intervention (1\%) was implemented in multiple countries across two continents (USA, Ecuador, Peru and Brazil) [84]. The risk behaviours that were targeted in various intervention countries, separated by studies with young adult male participants only and studies with outcomes stratified by age/ sex to include young adult males are shown in Table 2 . It is clear that the interventions targeting alcohol use were predominantly performed in the USA (84\%) and a similar pattern occurred for unsafe sexual behaviour (66.7\%). Although the USA was the leading intervention country in the other risk behaviours, the pattern tended to be less disproportionate.

\section{Study designs and risk behaviours}

Of the 100 included studies there were 62 (62\%) randomized controlled trials [51-57,59,62-73,75,77,78,82, 84-86,90,92,93,96,103,105,108,109,112,113,116,118-120, 123-125,127-131,133-135,138-140,143-149], 16 (16\%) case series $[57,60,61,74,76,80,91,94,99-101,110,115,117$, 121,136], 9 (9\%) non-randomized controlled trials [87, 95,98,102,104,107,122,137,141], 7 (7\%) cluster randomized controlled trials $[79,81,83,111,126,132,142], 4$ (4\%) cluster controlled studies $[45,88,97,114]$ and $2(2 \%)$ interrupted time series $[89,106]$. The number of papers for each study design and reported risk behaviour is provided in Table 3 . The randomized controlled design had the greatest percentage use in the physical inactivity interventions $(81.5 \%)$, whilst the unsafe sexual behaviour interventions had the greatest diversity in study design.

\section{Intervention setting and risk behaviours}

Interventions were implemented across a wide variety of settings with the most common being university/college settings $(\mathrm{n}=46,46 \%) \quad[53-59,62-66,68,70,73,75,78,93,95$, 96,98-101,103,105,108,109,112,115,117,118,121,123-126, $129,130,134,136,137,141,144,146,147]$. Other prevalent settings included military/army $(\mathrm{n}=11,11 \%)[52,60,61$, $69,71,72,104,110,119,127,128]$ and internet $(n=8,8 \%)$ $[45,85,92,113,132,139,148,149]$. Five studies (5\%) were delivered in multiple settings $[83,111,120,122,145]$. The risk behaviours across the different settings are summarised in Table 4. The university/college setting was the most prevalent in the physical inactivity interventions (63\%). This was also the leading setting (or equal leading) for seven out of the eight risk behaviours that were intervened upon and amongst the studies targeting multiple risk behaviours. All of the studies that utilized a military/army setting included young adult male participants only.

\section{Intervention mode and risk behaviours}

The included studies most commonly used 'face-to-face' delivery $(\mathrm{n}=71,71 \%)$ [51,53-58,60-82,84,86-91,94,96-105, 107-110,112,114,117,121,123-130,133-136,144,146,147]. Other modes included online $(\mathrm{n}=8,8 \%)$ [45,52,85, $113,132,139,148,149]$, paper $(\mathrm{n}=5,5 \%)[137,138,140,142$, 143], TV/video/DVD $(\mathrm{n}=2,2 \%)$ [92,93] and a social marketing campaign $(\mathrm{n}=1,1 \%)$ [106]. Many studies $(\mathrm{n}=$ 13, 13\%) implemented multi-faceted intervention modes $[59,83,95,111,115,116,118-120,122,131,141,145]$. The delivery mode for each risk behaviour is shown in Table 5, with the face-to-face the predominant approach across all risk behaviours. This mode also featured most commonly (either alone or with other intervention mode(s)) in physical inactivity studies (92.6\%). The use of technology based intervention modes (e.g. online or mobile phone) either alone or in combination with other mode(s) was less likely to be used in studies that included young adult male participants only.

\section{Sex of the Intervention facilitator and risk behaviours}

For those interventions delivered by individuals $(n=87)$, we examined who had delivered the intervention. Only 12 studies indicated the sex of the facilitator; of which 7 (7\%) were delivered by males only $[80,82,88,89,94,98$, $146]$, whilst $4(4 \%)$ of the studies included personnel with mixed sexes $[59,87,97,144]$ and one study delivered by a female [129]. For a breakdown of delivery personnel sex by risk behaviour refer to Table 6 . The use of a male facilitator was most common for unsafe sexual behaviour interventions $(28.6 \%)$ and also more common in the studies with only young adult male participants (13\%).

\section{Profession of the intervention facilitator and risk behaviours}

Most often a member of the research team $(n=20,20 \%)$ [54,57,58,62,63,86,94,99-101,104,107,109,112,134,136-139, 143], personal trainer/training supervisors $(n=8,8 \%)$ $[56,68,71-74,77,78]$, educated supervisors/trained facilitators $(\mathrm{n}=7,7 \%)[60,61,90,93,97,105,145]$ and counsellors $(\mathrm{n}=7,7 \%)[59,111,117,118,127,128,131]$ were involved in the delivery. In addition, interventions regularly incorporated multiple professionals for delivery $(n=9,9 \%)$ [75,80,81,83,87-89,125,144]. There were 20 (20\%) studies that failed to state the profession $[51,53,55,64-67,69,70$, $76,79,84,102,108,114,115,124,126,130,135]$ and $12(12 \%)$ of the studies were self-directed [45,52,85,91,106,113, $120,132,140,142,148,149]$. A summary of the risk behaviours and by whom the intervention was delivered by is available in Table 7. The profession of the facilitator tended to be diverse across the risk behaviours. Failing 
Table 2 Intervention country by risk behaviours

\begin{tabular}{|c|c|c|c|c|c|c|c|c|c|c|c|c|}
\hline Intervention country & $\begin{array}{l}\text { Physical } \\
\text { inactivity }\end{array}$ & $\begin{array}{c}\text { Poor } \\
\text { diet }\end{array}$ & $\begin{array}{l}\text { Alcohol } \\
\text { use }\end{array}$ & $\begin{array}{l}\text { Tobacco } \\
\text { smoking }\end{array}$ & $\begin{array}{l}\text { Recreational } \\
\text { drug use }\end{array}$ & $\begin{array}{l}\text { Unsafe sexual } \\
\text { behaviour }\end{array}$ & Violence & $\begin{array}{l}\text { Unsafe } \\
\text { vehicle } \\
\text { driving }\end{array}$ & Gambling & $\begin{array}{c}\text { Tanning/sun } \\
\text { exposure }\end{array}$ & $\begin{array}{l}\text { Self- } \\
\text { harm }\end{array}$ & $\begin{array}{c}\text { Multiple risk } \\
\text { behaviours }\end{array}$ \\
\hline
\end{tabular}

Included young adult male

participants only

USA

UK

Australia

Brazil

Turkey

Finland

Iran

Israel

South Africa

Thailand

France

Switzerland

Sweden

Canada

Total

Studies with outcomes stratified

by age and/or sex to include

young adult males

USA

Australia

Brazil

Turkey

Israel

Mexico

Denmark

Netherlands

New Zealand

China

Italy

Sweden 
Table 2 Intervention country by risk behaviours (Continued)

\begin{tabular}{|c|c|c|c|c|c|c|c|c|c|c|c|c|c|}
\hline Peru & 0 & 0 & 0 & 0 & 0 & 1 & 0 & 0 & 0 & 0 & 0 & 0 & 1 \\
\hline $\begin{array}{l}\text { International (multiple } \\
\text { countries) } \$ \S\end{array}$ & 0 & 0 & 0 & 0 & 0 & 1 & 0 & 0 & 0 & 0 & 0 & 0 & 1 \\
\hline Total & 9 & 4 & 21 & 3 & 2 & 7 & 0 & 3 & 0 & 0 & 0 & 5 & 54 \\
\hline Overall total & 27 & 5 & 25 & 4 & 2 & 21 & 0 & 5 & 0 & 1 & 0 & 10 & 100 \\
\hline
\end{tabular}

${ }^{*}$ Poor diet and Physical inactivity.

Alcohol use and Recreational drug use.

Alcohol use and Unsafe sexual behaviour.

**** Poor diet, physical inactivity and tobacco smoking.

Poor diet, physical inactivity and tobacco

Alcohol use and physical inactivity.
${ }^{\ddagger}$ Unsafe vehicle driving and alcohol use.

ST Unafe vehicle diving and alcohol use.

$\$ \$$ Countries included: USA. Ecuador, Peru and Brazil. 
Table 3 Study design by risk behaviours

\begin{tabular}{|c|c|c|c|c|c|c|c|c|c|c|c|c|c|}
\hline Study design & $\begin{array}{l}\text { Physical } \\
\text { inactivity }\end{array}$ & $\begin{array}{l}\text { Poor } \\
\text { diet }\end{array}$ & $\begin{array}{l}\text { Alcohol } \\
\text { use }\end{array}$ & $\begin{array}{l}\text { Tobacco } \\
\text { smoking }\end{array}$ & $\begin{array}{l}\text { Recreational } \\
\text { drug use }\end{array}$ & $\begin{array}{c}\text { Unsafe sexual } \\
\text { behaviour }\end{array}$ & Violence & $\begin{array}{l}\text { Unsafe vehicle } \\
\text { driving }\end{array}$ & Gambling & $\begin{array}{c}\text { Tanning/sun } \\
\text { exposure }\end{array}$ & $\begin{array}{l}\text { Self- } \\
\text { harm }\end{array}$ & $\begin{array}{c}\text { Multiple risk } \\
\text { behaviours }\end{array}$ & Total \\
\hline \multicolumn{14}{|c|}{$\begin{array}{l}\text { Included young adult } \\
\text { male participants only }\end{array}$} \\
\hline RCT & 14 & 1 & 3 & 0 & 0 & 6 & 0 & 1 & 0 & 0 & 0 & $4^{* * * * *}, \S, 1, \neq$ & 29 \\
\hline Non- RCT & 0 & 0 & 0 & 0 & 0 & 2 & 0 & 1 & 0 & 0 & 0 & $1^{*}$ & 4 \\
\hline Case series & 4 & 0 & 0 & 1 & 0 & 3 & 0 & 0 & 0 & 1 & 0 & 0 & 9 \\
\hline Cluster RCT & 0 & 0 & 1 & 0 & 0 & 0 & 0 & 0 & 0 & 0 & 0 & 0 & 1 \\
\hline $\begin{array}{l}\text { Cluster } \\
\text { controlled }\end{array}$ & 0 & 0 & 0 & 0 & 0 & 2 & 0 & 0 & 0 & 0 & 0 & 0 & 2 \\
\hline $\begin{array}{l}\text { Interrupted } \\
\text { time series }\end{array}$ & 0 & 0 & 0 & 0 & 0 & 1 & 0 & 0 & 0 & 0 & 0 & 0 & 1 \\
\hline Total & 18 & 1 & 4 & 1 & 0 & 14 & 0 & 2 & 0 & 1 & 0 & 5 & 46 \\
\hline \multicolumn{14}{|c|}{$\begin{array}{l}\text { Studies with outcomes } \\
\text { stratified by age and/or } \\
\text { sex to include young } \\
\text { adult males }\end{array}$} \\
\hline RCT & 8 & 0 & 15 & 2 & 1 & 3 & 0 & 1 & 0 & 0 & 0 & $3^{* * *}, * * *$ & 33 \\
\hline Non- RCT & 0 & 1 & 2 & 0 & 0 & 1 & 0 & 1 & 0 & 0 & 0 & 0 & 5 \\
\hline Case series & 1 & 3 & 1 & 0 & 0 & 017 & 0 & 0 & 0 & 0 & 0 & $2^{* * * * *}$ & 7 \\
\hline Cluster RCT & 0 & 0 & 2 & 1 & 0 & 3 & 0 & 0 & 0 & 0 & 0 & 0 & 6 \\
\hline $\begin{array}{l}\text { Cluster } \\
\text { controlled }\end{array}$ & 0 & 0 & 1 & 0 & 1 & 0 & 0 & 0 & 0 & 0 & 0 & 0 & 2 \\
\hline $\begin{array}{l}\text { Interrupted } \\
\text { time series }\end{array}$ & 0 & 0 & 0 & 0 & 0 & 0 & 0 & 1 & 0 & 0 & 0 & 0 & 1 \\
\hline Total & 9 & 4 & 21 & 3 & 2 & 7 & 0 & 3 & 0 & 0 & 0 & 5 & 54 \\
\hline Overall total & 27 & 5 & 25 & 4 & 2 & 21 & 0 & 5 & 0 & 1 & 0 & 10 & 100 \\
\hline
\end{tabular}

${ }_{* * *}^{*}$ Poor diet and Physical inactivity.

Alcohol use and Recreational drug use.

*** Alcohol use and Unsafe sexual behaviour.

Poor diet, physical inactivity and tobacco smoking.

${ }^{\dagger}$ Alcohol use and physical inactivity.

₹ Unsafe vehicle driving and alcohol use.

${ }^{\mathrm{s}}$ Tobacco smoking, alcohol use and recreational drug use. 
Table 4 Intervention setting by risk behaviours

\begin{tabular}{|c|c|c|c|c|c|c|c|c|c|c|c|c|c|}
\hline Intervention setting & $\begin{array}{l}\text { Physical } \\
\text { inactivity }\end{array}$ & $\begin{array}{l}\text { Poor } \\
\text { diet }\end{array}$ & $\begin{array}{l}\text { Alcohol } \\
\text { use }\end{array}$ & $\begin{array}{l}\text { Tobacco } \\
\text { smoking }\end{array}$ & $\begin{array}{l}\text { Recreational } \\
\text { drug use }\end{array}$ & $\begin{array}{c}\text { Unsafe sexual } \\
\text { behaviour }\end{array}$ & Violence & $\begin{array}{l}\text { Unsafe } \\
\text { vehicle } \\
\text { driving }\end{array}$ & Gambling & $\begin{array}{l}\text { Tanning/sun } \\
\text { exposure }\end{array}$ & $\begin{array}{l}\text { Self- } \\
\text { harm }\end{array}$ & $\begin{array}{c}\text { Multiple risk } \\
\text { behaviours }\end{array}$ & Total \\
\hline
\end{tabular}

Included young adult male

participants only

University/ college

Community

Primary- care setting

Military/army

Prison

Exercise laboratory

Internet

Home

Primary care + gym

Not stated

Total

Studies with outcomes stratified

by age and/or sex to include

young adult males

University/ college

School

Workplace

Community 
Table 4 Intervention setting by risk behaviours (Continued)

\begin{tabular}{|c|c|c|c|c|c|c|c|c|c|c|c|c|c|}
\hline Primary- care setting & 0 & 0 & 0 & 0 & 0 & 1 & 0 & 0 & 0 & 0 & 0 & 0 & 1 \\
\hline Hospital & 0 & 0 & 2 & 0 & 0 & 0 & 0 & 0 & 0 & 0 & 0 & 0 & 2 \\
\hline Exercise laboratory & 1 & 0 & 0 & 0 & 0 & 0 & 0 & 0 & 0 & 0 & 0 & 0 & 1 \\
\hline Internet & 0 & 0 & 5 & 0 & 1 & 1 & 0 & 0 & 0 & 0 & 0 & 0 & 7 \\
\hline Home & 0 & 1 & 4 & 0 & 0 & 0 & 0 & 0 & 0 & 0 & 0 & 0 & 5 \\
\hline Car & 0 & 0 & 0 & 0 & 0 & 0 & 0 & 1 & 0 & 0 & 0 & 0 & 1 \\
\hline Internet + home & 0 & 0 & 0 & 0 & 0 & 0 & 0 & 0 & 0 & 0 & 0 & $1^{*}$ & 1 \\
\hline Internet + University/college & 0 & 0 & 1 & 1 & 0 & 0 & 0 & 0 & 0 & 0 & 0 & 0 & 2 \\
\hline Community + Primary care & 0 & 0 & 0 & 0 & 0 & 1 & 0 & 0 & 0 & 0 & 0 & 0 & 1 \\
\hline Total & 9 & 4 & 21 & 3 & 2 & 7 & 0 & 3 & 0 & 0 & 0 & 0 & 54 \\
\hline Overall total & 27 & 5 & 25 & 4 & 2 & 21 & 0 & 5 & 0 & 1 & 0 & 10 & 100 \\
\hline
\end{tabular}

* Poor diet and Physical inactivity.

*** Alcohol use and Recreational drug use.

A*** Alcohol use and Unsafe sexual behaviour.

***** Poor diet, physical inactivity and tobacco smoking.

Alcohol use and physical inactivity.

₹ Unsafe vehicle driving and alcohol use.

${ }^{5}$ Tobacco smoking, alcohol use and recreational drug use. 
Table 5 Intervention mode by risk behaviour

\begin{tabular}{|c|c|c|c|c|c|c|c|c|c|c|c|c|c|}
\hline Intervention mode & $\begin{array}{l}\text { Physical } \\
\text { inactivity }\end{array}$ & $\begin{array}{l}\text { Poor } \\
\text { diet }\end{array}$ & $\begin{array}{l}\text { Alcohol } \\
\text { use }\end{array}$ & $\begin{array}{l}\text { Tobacco } \\
\text { smoking }\end{array}$ & $\begin{array}{l}\text { Recreational } \\
\text { drug use }\end{array}$ & $\begin{array}{c}\text { Unsafe sexual } \\
\text { behaviour }\end{array}$ & Violence & $\begin{array}{l}\text { Unsafe } \\
\text { vehicle } \\
\text { driving }\end{array}$ & Gambling & $\begin{array}{l}\text { Tanning/sun } \\
\text { exposure }\end{array}$ & $\begin{array}{l}\text { Self- } \\
\text { harm }\end{array}$ & $\begin{array}{c}\text { Multiple risk } \\
\text { behaviour }\end{array}$ & Total \\
\hline \multicolumn{14}{|c|}{$\begin{array}{l}\text { Included young adult male } \\
\text { participants only }\end{array}$} \\
\hline Online & 1 & 0 & 0 & 0 & 0 & 1 & 0 & 0 & 0 & 0 & 0 & 0 & 2 \\
\hline Face to face & 17 & 1 & 4 & 1 & 0 & 13 & 0 & 2 & 0 & 1 & 0 & $2^{+, \neq}$ & 41 \\
\hline Face-to-Face + Paper & 0 & 0 & 0 & 0 & 0 & 0 & 0 & 0 & 0 & 0 & 0 & $2^{*, * * * *}$ & 2 \\
\hline $\begin{array}{l}\text { Face-to Face + mobile } \\
\text { phone }\end{array}$ & 0 & 0 & 0 & 0 & 0 & 0 & 0 & 0 & 0 & 0 & 0 & $1^{\S}$ & 1 \\
\hline Total & 18 & 1 & 4 & 1 & 0 & 14 & 0 & 2 & 0 & 1 & 0 & 5 & 46 \\
\hline
\end{tabular}

Studies with outcomes stratified

by age and/or sex to include

young adult males

Online

Paper

Face to face

TV/Video/DVD

Social marketing

Face-to-Face + Online +

Mobile phone

Face-to-Face + Paper

Online + Mobile phone

Face-to Face + mobile

phone

Face- to- Face + Online

Total

Overall total

Poor diet and Physical inactivity.

Alcohol use and Recreational drug use.

Alcohol use and Unsafe sexual behaviour.

Poor diet, physical inactivity and tobacco smoking.

${ }^{\dagger}$ Alcohol use and physical inactivity.

* Unsafe vehicle driving and alcohol use.

Tobacco smoking, alcohol use and recreational drug use. 
Table 6 Sex of the intervention facilitator and risk behaviours

\begin{tabular}{|c|c|c|c|c|c|c|c|c|c|c|c|c|c|}
\hline $\begin{array}{l}\text { Intervention } \\
\text { delivered by }\end{array}$ & $\begin{array}{l}\text { Physical } \\
\text { inactivity }\end{array}$ & $\begin{array}{l}\text { Poor } \\
\text { diet }\end{array}$ & $\begin{array}{l}\text { Alcohol } \\
\text { use }\end{array}$ & $\begin{array}{l}\text { Tobacco } \\
\text { smoking }\end{array}$ & $\begin{array}{l}\text { Recreational } \\
\text { drug use }\end{array}$ & $\begin{array}{c}\text { Unsafe sexual } \\
\text { behaviour }\end{array}$ & Violence & $\begin{array}{c}\text { Unsafe vehicle } \\
\text { driving }\end{array}$ & Gambling & $\begin{array}{c}\text { Tanning/sun } \\
\text { exposure }\end{array}$ & $\begin{array}{l}\text { Self- } \\
\text { harm }\end{array}$ & $\begin{array}{c}\text { Multiple risk } \\
\text { behaviour }\end{array}$ & Total \\
\hline \multicolumn{14}{|c|}{$\begin{array}{l}\text { Included young adult } \\
\text { male participants only }\end{array}$} \\
\hline Male only & 0 & 0 & 0 & 0 & 0 & 6 & 0 & 0 & 0 & 0 & 0 & 0 & 6 \\
\hline Female only & 0 & 0 & 1 & 0 & 0 & 0 & 0 & 0 & 0 & 0 & 0 & 0 & 1 \\
\hline Mixed & 0 & 0 & 0 & 0 & 0 & 2 & 0 & 0 & 0 & 0 & 0 & 0 & 2 \\
\hline Not stated & 17 & 1 & 3 & 1 & 0 & 4 & 0 & 2 & 0 & 1 & 0 & $5^{*}, * * * *,+, \neq, \S$ & 34 \\
\hline$N / A$ & 1 & 0 & 0 & 0 & 0 & 2 & 0 & 0 & 0 & 0 & 0 & 0 & 3 \\
\hline Total & 18 & 1 & 4 & 1 & 0 & 14 & 0 & 2 & 0 & 1 & 0 & 5 & 46 \\
\hline \multicolumn{14}{|c|}{$\begin{array}{l}\text { Studies with outcomes } \\
\text { stratified by age and/or } \\
\text { sex to include young } \\
\text { adult males }\end{array}$} \\
\hline Male only & 0 & 0 & 1 & 0 & 0 & 0 & 0 & 0 & 0 & 0 & 0 & 0 & 1 \\
\hline Female only & 0 & 0 & 0 & 0 & 0 & 0 & 0 & 0 & 0 & 0 & 0 & 0 & 0 \\
\hline Mixed & 1 & 0 & 1 & 0 & 0 & 0 & 0 & 0 & 0 & 0 & 0 & 0 & 2 \\
\hline Not stated & 8 & 4 & 13 & 3 & 1 & 6 & 0 & 2 & 0 & 0 & 0 & $4^{*}, * *, * *, * * * *$ & 41 \\
\hline$N / A$ & 0 & 0 & 6 & 0 & 1 & 1 & 0 & 1 & 0 & 0 & 0 & $1^{*}$ & 10 \\
\hline Total & 9 & 4 & 21 & 3 & 2 & 7 & 0 & 3 & 0 & 0 & 0 & 5 & 54 \\
\hline Overall total & 27 & 5 & 25 & 4 & 2 & 21 & 0 & 5 & 0 & 1 & 0 & 10 & 100 \\
\hline
\end{tabular}


Table 7 Profession of the intervention facilitator and risk behaviours

\begin{tabular}{|c|c|c|c|c|c|c|c|c|c|c|c|c|c|}
\hline Intervention delivered by & $\begin{array}{l}\text { Physical } \\
\text { inactivity }\end{array}$ & $\begin{array}{l}\text { Poor } \\
\text { diet }\end{array}$ & $\begin{array}{l}\text { Alcohol } \\
\text { use }\end{array}$ & $\begin{array}{l}\text { Tobacco } \\
\text { smoking }\end{array}$ & $\begin{array}{l}\text { Recreational } \\
\text { drug use }\end{array}$ & $\begin{array}{l}\text { Unsafe sexual } \\
\text { behaviour }\end{array}$ & Violence & $\begin{array}{c}\text { Unsafe } \\
\text { vehicle driving }\end{array}$ & Gambling & $\begin{array}{l}\text { Tanning/sun } \\
\text { exposure }\end{array}$ & $\begin{array}{l}\text { Self- } \\
\text { harm }\end{array}$ & $\begin{array}{c}\text { Multiple risk } \\
\text { behaviour }\end{array}$ & Total \\
\hline \multicolumn{14}{|l|}{$\begin{array}{l}\text { Included young adult male } \\
\text { participants only }\end{array}$} \\
\hline Doctor/physician/clinician & 0 & 0 & 0 & 0 & 0 & 0 & 0 & 0 & 0 & 0 & 0 & $1^{* * * *}$ & 1 \\
\hline Dietician & 0 & 1 & 0 & 0 & 0 & 0 & 0 & 0 & 0 & 0 & 0 & $1^{*}$ & 2 \\
\hline Nurse & 0 & 0 & 0 & 1 & 0 & 0 & 0 & 0 & 0 & 0 & 0 & 0 & 1 \\
\hline Member of research team & 3 & 0 & 0 & 0 & 0 & 2 & 0 & 1 & 0 & 0 & 0 & 0 & 6 \\
\hline $\begin{array}{l}\text { Personal trainer/ training } \\
\text { supervisor }\end{array}$ & 4 & 0 & 0 & 0 & 0 & 0 & 0 & 0 & 0 & 0 & 0 & 0 & 4 \\
\hline $\begin{array}{l}\text { Educated supervisor/ Trained } \\
\text { facilitators }\end{array}$ & 2 & 0 & 0 & 0 & 0 & 2 & 0 & 1 & 0 & 0 & 0 & 0 & 5 \\
\hline Health educator/advisor & 0 & 0 & 0 & 0 & 0 & 1 & 0 & 0 & 0 & 0 & 0 & 0 & 1 \\
\hline Peer educators & 0 & 0 & 0 & 0 & 0 & 2 & 0 & 0 & 0 & 0 & 0 & 0 & 2 \\
\hline Counsellor & 0 & 0 & 2 & 0 & 0 & 0 & 0 & 0 & 0 & 0 & 0 & 0 & 2 \\
\hline Psychologist & 0 & 0 & 1 & 0 & 0 & 0 & 0 & 0 & 0 & 0 & 0 & $1^{\S}$ & 2 \\
\hline Self-learn & 1 & 0 & 0 & 0 & 0 & 2 & 0 & 0 & 0 & 0 & 0 & 0 & 3 \\
\hline $\begin{array}{l}\text { Health educators + social worker } \\
+ \text { Doctor/physician/clinician }\end{array}$ & 0 & 0 & 0 & 0 & 0 & 1 & 0 & 0 & 0 & 0 & 0 & 0 & 1 \\
\hline $\begin{array}{l}\text { Peer educators + community } \\
\text { advisory board }\end{array}$ & 0 & 0 & 0 & 0 & 0 & 2 & 0 & 0 & 0 & 0 & 0 & 0 & 2 \\
\hline $\begin{array}{l}\text { Doctor/physician/clinician + } \\
\text { Nurses + Health educators }\end{array}$ & 0 & 0 & 0 & 0 & 0 & 1 & 0 & 0 & 0 & 0 & 0 & 0 & 1 \\
\hline $\begin{array}{l}\text { Trained facilitator }+ \text { Nurse }+ \\
\text { Meditator }\end{array}$ & 0 & 0 & 0 & 0 & 0 & 0 & 0 & 0 & 0 & 0 & 0 & $1^{\dagger}$ & 1 \\
\hline Not stated & 8 & 0 & 1 & 0 & 0 & 1 & 0 & 0 & 0 & 1 & 0 & $1^{\ddagger}$ & 12 \\
\hline Total & 18 & 1 & 4 & 1 & 0 & 14 & 0 & 2 & 0 & 1 & 0 & 5 & 46 \\
\hline \multicolumn{14}{|c|}{$\begin{array}{l}\text { Studies with outcomes stratified by age } \\
\text { and/or sex to include young adult males }\end{array}$} \\
\hline Student & 0 & 0 & 3 & 0 & 0 & 0 & 0 & 0 & 0 & 0 & 0 & $1^{* *}$ & 4 \\
\hline Member of research team & 2 & 3 & 6 & 2 & 0 & 0 & 0 & 1 & 0 & 0 & 0 & 0 & 14 \\
\hline $\begin{array}{l}\text { Personal trainer/ training } \\
\text { supervisor }\end{array}$ & 4 & 0 & 0 & 0 & 0 & 0 & 0 & 0 & 0 & 0 & 0 & 0 & 4 \\
\hline $\begin{array}{l}\text { Educated supervisor/ Trained } \\
\text { facilitators }\end{array}$ & 0 & 0 & 1 & 0 & 0 & 1 & 0 & 0 & 0 & 0 & 0 & 0 & 2 \\
\hline Peer educators & 0 & 0 & 0 & 0 & 0 & 1 & 0 & 0 & 0 & 0 & 0 & 0 & 1 \\
\hline Counsellor & 1 & 0 & 1 & 1 & 0 & 0 & 0 & 0 & 0 & 0 & 0 & $2^{* *}, * * * *$ & 5 \\
\hline Psychologist & 0 & 0 & 0 & 0 & 0 & 0 & 0 & 0 & 0 & 0 & 0 & $1^{*}$ & 1 \\
\hline
\end{tabular}


Table 7 Profession of the intervention facilitator and risk behaviours (Continued)

\begin{tabular}{|c|c|c|c|c|c|c|c|c|c|c|c|c|c|}
\hline Social Worker & 0 & 0 & 1 & 0 & 0 & 0 & 0 & 0 & 0 & 0 & 0 & 0 & 1 \\
\hline Self-learn & 0 & 0 & 6 & 0 & 1 & 0 & 0 & 1 & 0 & 0 & 0 & $1^{*}$ & 9 \\
\hline $\begin{array}{l}\text { Member of research team + } \\
\text { student }\end{array}$ & 1 & 0 & 0 & 0 & 0 & 0 & 0 & 0 & 0 & 0 & 0 & 0 & 1 \\
\hline Psychologist + Student & 0 & 0 & 1 & 0 & 0 & 0 & 0 & 0 & 0 & 0 & 0 & 0 & 1 \\
\hline $\begin{array}{l}\text { Doctor/physician/clinician + Peer } \\
\text { educators }\end{array}$ & 0 & 0 & 0 & 0 & 0 & 1 & 0 & 0 & 0 & 0 & 0 & 0 & 1 \\
\hline Teachers + Peer educators & 0 & 0 & 0 & 0 & 0 & 1 & 0 & 0 & 0 & 0 & 0 & 0 & 1 \\
\hline Not stated & 1 & 1 & 2 & 0 & 1 & 2 & 0 & 1 & 0 & 0 & 0 & 0 & 8 \\
\hline N/A & 0 & 0 & 0 & 0 & 0 & 1 & 0 & 0 & 0 & 0 & 0 & 0 & 1 \\
\hline Total & 9 & 4 & 21 & 3 & 2 & 7 & 0 & 3 & 0 & 0 & 0 & 5 & 54 \\
\hline Overall total & 27 & 5 & 25 & 4 & 2 & 21 & 0 & 5 & 0 & 1 & 0 & 10 & 100 \\
\hline
\end{tabular}

${ }^{*}$ Poor diet and Physical inactivity.

***ohol use and Recreational drug use.

Alcohol use and Unsafe sexual behaviour.

*** Poor diet, physical inactivity and tobacco smoking.

${ }^{\dagger}$ Alcohol use and physical inactivity.

* Unsafe vehicle driving and alcohol use.

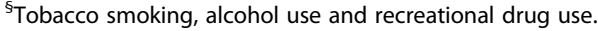


to report the profession of the facilitator was mainly done in the physical inactivity studies (33.3\%).

\section{Theoretical framework and risk behaviours}

Only 19 studies (19\%) reported utilising a theoretical framework to develop the intervention. The transtheoretical model was most commonly used $(\mathrm{n}=4,4 \%)$ $[52,110,112,121]$, followed by social cognitive theory $(\mathrm{n}=3,3 \%)[81,100,101]$. In addition several studies $(\mathrm{n}=7$, 7\%) used multiple frameworks to help with development of the interventions $[45,59,75,90,111,114,132]$. A summary of the theoretical frameworks utilized, by risk behaviours, is summarised in Table 8 . The most commonly used theoretical framework (either alone or with another framework/model) was the trans-theoretical model (10\%). The risk behaviour that was most likely to report use of a theoretical framework was for poor diet (40\%). Contrary to this, failure to report use of a theoretical framework was most common in unsafe vehicle driving interventions (100\%).

\section{Discussion}

The aim of this scoping review was to provide a broad overview of interventions that have targeted risk behaviours in young adult males aged 17 to 35 years. Although the search strategy identified over 16,000 articles, the final number of studies that only included young adult male participants was low $(\mathrm{n}=46)$. The remaining studies $(\mathrm{n}=54)$ stratified and reported outcomes by age and/or sex. The three risk behaviours most frequently targeted in interventions that have been conducted in young adult males to date were: physical inactivity $(\mathrm{n}=27)$, alcohol use $(n=25)$, and unsafe sexual behaviours $(n=21)$. These three risk behaviours were also targeted most frequently in studies that included only young adult male participants (physical inactivity $\mathrm{n}=18$, unsafe sexual behaviour $\mathrm{n}=14$ and alcohol use $n=4$ ). In comparison, there were smaller numbers of studies targeting poor diet, recreational drug use, tobacco smoking, unsafe vehicle driving and tanning/ sun exposure, with no relevant studies targeting gambling, violence or self-harm.

The distribution of risk behaviours within the included interventions mostly corresponds with prevalence rates from USA-based studies. This and the heterogeneity in reporting makes it difficult to draw conclusions. Low fruit and vegetable intakes, alcohol use and obesity rates were the most prevalent risk behaviours in young men in the USA. Although this scoping review identified few interventions on poor diet in young men, there were greater numbers of interventions for physical inactivity. Given the high prevalence of alcohol use young men in the USA and that alcohol is the leading contributor to lost DALYs (7.4\% of total DALYs) in males it is not surprising that a high proportion of interventions targeting alcohol use were identified in this review.
Few interventions included young adult male participants only $(\mathrm{n}=46)$. Most studies targeted young adults in general (male and female) $(\mathrm{n}=51)$ in the same intervention and then stratified results by sex. However, stratifying the results by sex and examining the sex comparisons for certain parameters can often obscure the within- and across sex variations which can limit the accuracy and applicability of results [31]. In addition the European commission for men's health have highlighted that the biological and gender behaviour differences represent different needs and perceived barriers in terms of health promotion and intervention design [29]. Consequently, designing interventions for both sexes together is not likely to suit all characteristics of either sex, and may disregard one over the other or fail to engage both. Predominantly studies in this review applied a 'one size fits all approach' which may be detrimental to both men and women [29]. Tailored interventions can nullify these limitations, taking into account the social diversity that is apparent in young men and making applicable for all the different subgroups (e.g. fathers, unemployed, students, homosexual men, ethnic minorities) [35]. Previous research has shown gender tailoring can be effective in men [150,151].

Early work has suggested hegemonic masculinity to be a strong predictor of risky behaviours [30] and is linked with poorer health behaviours [24-26]. However modern societal changes suggest a move away from the conventional hegemonic masculinity and a move towards a plurality of masculinities [152]. Masculinity is not a fixed entity and can change according to gender relations in a particular setting [153]. According to Mullen et al [152] young men are constructing post-modern masculine identities because of changes to youth culture, leisure patterns, employment (i.e. fewer job opportunities) and educational destinations (i.e. more young men in vocational and higher education). Therefore future research should not treat young men as one homogenous group, particularly because they are going through a range of key life events such as moving away from home [23], developing emerging sexual identities [152], starting work, parenthood and unemployment [152]. Further, this supports the need for tailored interventions specific to the unique characteristics of the individual person and by taking into account the social diversity across young men.

Theoretical frameworks are useful during the planning, implementing and evaluating interventions [154] and can maximise the chance of success, with increases in effect size for health-related behaviours including physical inactivity, poor diet, tobacco smoking and alcohol use [155]. Most of the interventions failed to list or operationalise theories. Future interventions targeting these risk behaviours should consider framing and operationalising appropriate theories to improve likelihood 
Table 8 Theoretical framework and risk behaviour

\begin{tabular}{|c|c|c|c|c|c|c|c|c|c|c|c|c|c|}
\hline Theoretical framework & $\begin{array}{l}\text { Physical } \\
\text { inactivity }\end{array}$ & $\begin{array}{c}\text { Poor } \\
\text { diet }\end{array}$ & $\begin{array}{l}\text { Alcohol } \\
\text { use }\end{array}$ & $\begin{array}{l}\text { Tobacco } \\
\text { smoking }\end{array}$ & $\begin{array}{l}\text { Recreational } \\
\text { drug use }\end{array}$ & $\begin{array}{c}\text { Unsafe } \\
\text { sexual behaviour }\end{array}$ & Violence & $\begin{array}{c}\text { Unsafe } \\
\text { vehicle driving }\end{array}$ & Gambling & $\begin{array}{c}\text { Tanning/ } \\
\text { sun exposure }\end{array}$ & $\begin{array}{l}\text { Self- } \\
\text { harm }\end{array}$ & $\begin{array}{c}\text { Multiple } \\
\text { risk behaviour }\end{array}$ & Total \\
\hline \multicolumn{14}{|l|}{$\begin{array}{l}\text { Included young adult male } \\
\text { participants only }\end{array}$} \\
\hline Trans-Theoretical Model & 1 & 0 & 0 & 1 & 0 & 0 & 0 & 0 & 0 & 0 & 0 & 0 & 2 \\
\hline $\begin{array}{l}\text { Integrated Model of } \\
\text { Behaviour Theory }\end{array}$ & 0 & 0 & 0 & 0 & 0 & 1 & 0 & 0 & 0 & 0 & 0 & 0 & 1 \\
\hline $\begin{array}{l}\text { Social Cognitive Theory + } \\
\text { Trans-Theoretical Model }\end{array}$ & 0 & 0 & 0 & 0 & 0 & 1 & 0 & 0 & 0 & 0 & 0 & 0 & 1 \\
\hline Not stated & 17 & 1 & 4 & 0 & 0 & 12 & 0 & 2 & 0 & 1 & 0 & $5^{* * * * * *},+, \neq, \S$ & 42 \\
\hline Total & 18 & 1 & 4 & 1 & 0 & 14 & 0 & 2 & 0 & 1 & 0 & 5 & 46 \\
\hline
\end{tabular}

Studies with outcomes stratified by age and/or sex to include young adult males

Social Cognitive Theory

Trans-Theoretical Model

Health Promotion Mode

Self-Regulation Theory

Theory of Cognitive

conditioning

Process of Change Theory

Social Cognitive Theory

Trans-Theoretical Model

Community Organization

Model + Behaviour Change

Model.

Trans-Theoretical Model +

Health Belief Model

Trans-Theoretical Model +

Health Belief Model + Theory

of Planned Behaviour

Not stated

Total

Overall total

$\begin{array}{ccccc}7 & 2 & 18 & 0 & 1 \\ 9 & 4 & 21 & 3 & 2 \\ 27 & 5 & 25 & 4 & 2\end{array}$

Poor diet and Physical inactivity.

Alcohol use and Recreational drug use.

Alcohol use and Unsafe sexual behaviour.

Poor diet, physical inactivity and tobacco smoking.

Alcohol use and physical inactivity.

Unsafe vehicle driving and alcohol use.

Tobacco smoking, alcohol use and recreational drug use. 
of success [156]. The Health, Illness, Men and Masculinities (HIMM) theoretical framework [157] considers men's health in a larger social context and considers the influence of masculinity across the life course. In addition, the Communities of Practice Framework [158] examines how identities are learned and reproduced within certain subgroups and communities (e.g. families, workplace, sports teams, fraternities etc.) and can help to understand the shifts and discrepancies in young men's health and risk behaviour practices. Although both frameworks are not directly constructed for young men, they do show promise and provide direction for health related interventions aimed at the social diversities apparent in this group. Although research has shown that theoretical frameworks are effective, there is some conjecture in the literature $[159,160]$. The inconsistency in effectiveness may explain the lack of theoretical frameworks utilized by the included studies in this review.

Included studies predominantly recruited participants from, and conducted interventions within, a university/ college setting. This is likely to be due to the large numbers of young adult males located in these settings and also where researchers are based. This therefore brings into question the generalizability of the interventions and representativeness of the samples. University samples generally come from higher socio-economic backgrounds [161], therefore it is vital to recruit and engage a diverse population sample from a wide socio-economic and cultural demography. Developing interventions that take place in settings where young men usually congregate or are located may be effective in targeting a greater number of young adult males. This is particularly evident since all of the studies that utilised a military/army setting were exclusively young adult male samples and on several occasions sample sizes of $>500$ were achieved $[52,60,61,119,128]$.

The face-to-face method was the most common form of intervention delivery mode, even though this approach may be perceived as a high participant burden (e.g. associated time and travel factors) [162]. A small number of interventions used online and/or mobile phones as an intervention mode, with all of the studies using these methods published from 2008 onwards. However the best delivery mode for young men is unclear and research is required to determine reach, engagement and effect on outcomes.

Very few studies reported having male-only facilitators, even within male-only intervention studies. Early psychological research has emphasised the effects of similarity on attitude change and persuasion [163]. Facilitators who are similar to the participants in terms of sex may therefore influence intervention effectiveness. It is clear that people are more likely to disclose to others who are similar to them [164]. This may be most relevant to sexual behaviour interventions, given the sensitivity of this topic. This is supported by the greater number of male only facilitators in interventions addressing this risk behaviour in the current review and the greater number of peer-led educators. This factor may be an important element in the delivery of interventions for young men; however it was not addressed by the majority of other studies in this review. Therefore, the sex of the facilitator involved in intervention delivery should be considered in future intervention design, and reporting of research. For the interventions with no face-to-face contact in which facilitators are not required (e.g. online research), it is important to ensure the program remains sensitive to unique characteristics of the target population. Men's only weight loss interventions developed by Morgan and colleagues [165,166] achieved this by using preferred language of men and relevant examples in intervention materials (online and paper based). Similar approaches can be applied in interventions for young men that do not use face-to-face contact, to ensure it remains credible, relatable and likeable for the young men [167].

This scoping review has several strengths, namely: a comprehensive search strategy was implemented across multiple databases and grey literature with no date restrictions. The use of multiple risk behaviours provided a thorough synthesis of the evidence base in young adult men. Also a detailed data extraction process relative to other published scoping reviews provided comprehensive information about the included studies. Although, included studies were limited to English language papers only, it has been found that English language publications in public health are over-represented with $96.5 \%$ of 210,433 public health publications in Europe being reported in English [168]. Thus it is likely that only a small minority of studies would have been missed.

There were potentially additional studies that included young males, but results were not stratified by age and/ or sex and thus we could not be certain that the population in these studies were comprised of the target group and therefore these studies were excluded. Some studies included age and sex in the regression models during analysis but were ultimately excluded for failing to specifically report intervention outcome effects in young adult males. Similarly, several studies $(n=109)$ were excluded as they did not provide the age range of the participants. To identify a large volume of literature, a broad age range (aged 17-35 years) was used to define young men. However, young men are not homogenous and thus different risk behaviours may be more prevalent at particular life stages [157]. For example, men who are in their 30's may be very different to men in their late teens/early $20 s^{\prime}$ in lifestyle factors affecting health such as; marital status, occupational status, housing environment, educational attainment and family circumstances [169]. Therefore future research should look to present results 
by age sub-groups to better reflect the diversity in risk behaviours among young men.

Qualitative research seeks to study social relations of human behaviour [170] and was therefore not included in this review as we were interested in the describing which risk behaviours had been addressed in young men and the details regarding the components of these interventions. However, such information can provide valuable information on perceptions of risk and usefulness/acceptability on interventions for this group. Furthermore studies were excluded if participants were from low or low-to-middle income countries because of the large heterogeneity between low-income/low-to-middle income and upper-middle/ high-income countries. However, this presumes there is no difference between income and cultural groups within upper-middle/high-income countries, which is not the case and is another limitation of this review. The use of numerical analysis for the scoping review means we cannot assert whether risk taking permeates multiple practices in young men, but there is a need for future research to examine this further. Although the scoping review study design can identify large volumes of literature, they do not appraise the quality of evidence, nor do they address 'synthesis', that is the relative weight of evidence in favour of effectiveness of any particular interventions [46]. The database of literature identified in a scoping review can serve as a foundation for more detailed systematic reviews [171] where the quality of evidence and synthesis can be explored.

\section{Conclusions}

Young men have been targeted to change risk behaviours to some extent, but this has not been comprehensive and is disproportionate across risk behaviours. Considering the health implications associated with young men's lifestyle, interventions are required that exclusively target the promotion of healthy behaviours or decrease in risky behaviours in this group.

\section{Recommendations for researchers}

- Targeted and tailored interventions specific to young adult males are required instead of a 'one size fits all approach'. Interventions must address young male's personalities and behaviours during intervention development and use appropriate theoretical frameworks.

- Future research should report mean age as well as the age range of all participants, even though this is not strictly advised in the CONSORT statement [172]. Including an age range is vital to enable a detailed indication of the sample for whom the intervention was developed.

- If research is stratified by sex, future research must provide sample sizes for both sexes.
- If using a diverse age range to define young men, results presented by age sub-groups will help to identify any heterogeneity in lifestyle behaviours that may be linked to risky behaviours.

- Better reporting of effects by sex is required and possibly a series of Individual Participant Data (IPD) meta-analyses of existing trials to build on the sex database and produce more reliable results [173].

- Future interventions targeting risk behaviours should consider recruiting representative samples given the vast majority to date are in university samples. Choosing environments where young males often congregate e.g. military/army or sporting clubs [174] and using a variety of settings may help to achieve this.

- Risk taking is understood as a male issue, whether there are benefits in including significant other males (i.e. father-son or couple dyad interventions) need to be considered in future research.

- The use of male only facilitators when working with young adult males should be considered and reported, given that this could be a factor affecting the intervention delivery. Researchers should also report the sex of the intervention facilitator.

\section{Additional files}

Additional file 1: Search strategy.

Additional file 2: Details of included studies.

Competing interests

The authors declare that they have no competing interests.

\section{Authors' contributions}

LMA contributed to the methodological design of the study including searching, screening, extracting studies and drafting of the manuscript. MJH, MER, PJM and CEC contributed to the methodological design of the review, screening of studies, and revision of the manuscript. All authors read and approved the final manuscript.

\section{Acknowledgements}

We would like to thank the student researcher (Katherine Brain) for helping with the retrieval of articles and for the reviewing of abstracts and full-text articles. Also we would like to thank the medical librarian (Debbie Booth) for helping to initiate the search strategy. L. M. Ashton undertook this research as a part requirement for the degree of PhD (Nutrition and Dietetics), The University of Newcastle, Australia. L. M. Ashton is supported by an International Postgraduate Award Scholarship. M J Hutchesson is supported by a Postdoctoral Research Fellowship from the National Heart Foundation of Australia (\#100177).

\section{Author details}

${ }^{1}$ School of Health Sciences, Faculty of Health and Medicine, Priority Research Centre for Physical Activity and Nutrition, University of Newcastle, Callaghan Campus, Newcastle, Australia. ${ }^{2}$ School of Education, Faculty of Education and Arts, Priority Research Centre for Physical Activity and Nutrition, University of Newcastle, Callaghan Campus, Newcastle, Australia.

Received: 24 March 2014 Accepted: 8 September 2014 Published: 16 September 2014 
References

1. Risk factors to health. In http://www.aihw.gov.au/risk-factors/

2. Young people: health risks and solutions. Fact sheet $N^{\circ} 345$. In http:// www.who.int/mediacentre/factsheets/fs345/en/.

3. Robinson J, Hetrick SE, Martin C: Preventing suicide in young people: systematic review. Aust New Zeal J Psychiatr 2011, 45(1):3-26.

4. Shaffer HJ, Hall MN, Vander Bilt J: Estimating the prevalence of disordered gambling behaviour in the United States and Canada: a research synthesis. Am J Public Health 1999, 89(9):1369-1376.

5. Fisher DE, Geller AC: Disproportionate burden of melanoma mortality in young us men the possible role of biology and behaviour. JAMA 2013, 149(8):903.

6. World Health Organization: Global Health Risks: Mortality and Burden of Disease Attributable to Selected Major Risks. Geneva: World Health Organization; 2009:1-70.

7. Johnson BT, Scott-Sheldon LA, Carey MP: Meta-synthesis of health behaviour change meta-analyses. Am J Public Health 2010, 100(11):2193.

8. Sexually transmitted infections. Fact sheet $\mathrm{N}^{\circ} 110$. In http://www.who.int/ mediacentre/factsheets/fs110/en/indexhtm.

9. World Health Organization: Global Status Report on Alcohol and Health Geneva, Switzerland: World Health Organization; 2011.

10. Obesity and overweight. Fact sheet $\mathrm{N}^{\circ} 311$. In http://www.who.int/ mediacentre/factsheets/fs311/en/.

11. Park MJ, Paul Mulye T, Adams SH, Brindis CD, Irwin CE Jr: The health status of young adults in the United States. J Adolesc Health 2006, 39(3):305-317.

12. Ogden CL, Carroll M, Kit B, Flegal K: Prevalence of obesity among adults: United States, 2011-2012. NCHS Data Brief 2013, 131:1-8.

13. Australian Health Survey: First results 2011-2012. In http://www.abs.gov. au/ausstats/abs@.nsf/Lookup/4364.0.55.001main+features12011-12.

14. Health inequalities: Adult obesity prevalence by age and sex. In http:// www.noo.org.uk/NOO_about_obesity/inequalities.

15. Bauman A, Bull F, Chey T, Craig CL, Ainsworth BE, Sallis JF, Bowles HR, Hagstromer M, Sjostrom M, Pratt M: The international prevalence study on physical activity: results from 20 countries. Int J Behav Nutr Phys Act 2009, 6:21.

16. Usual Dietary Intakes: Food Intakes, US Population, 2007-10. In. Applied Research Program Web site http://appliedresearch.cancer.gov/diet/ usualintakes/pop/2007-10/

17. Agaku IT, King BA, Dube SR: Current cigarette smoking among adultsUnited States, 2005-2012. MMWR Morb Mortal Wkly Rep 2014, 63(2):29-34

18. Chen $\mathrm{CM}$, Dufour MC, Yi H-Y: Alcohol consumption among young adults ages 18-24 in the United States: Results from the 2001-2002 NESARC Survey. Alcohol Res Health 2004, 28(4):269-281.

19. Cook RL, Clark DB: Is there an association between alcohol consumption and sexually transmitted diseases? A systematic review. Sex Transm Dis 2005, 32(3):156-164.

20. National Highway Traffic Safety Administration (NHTSA): Traffic Safety Facts 2003 Annual Report: Early Edition. Washington: US Department of Transportation; 2004

21. Bleyer A, O'Leary M, Barr R, Ries L: Cancer epidemiology in older adolescents and young adults 15 to 29 years of age, including SEER incidence and survival: 1975-2000. In Cancer Epidemiology in Older Adolescents and Young Adults 15 to 29 Years of Age, Including Seer Incidence and Survival: 1975-2000. 2006.

22. Centers for Disease Control and Prevention: A Report of the Surgeon General: Preventing Tobacco Use Among Youth and Young Adults. ; 2012:1-20.

23. Poobalan AS, Aucott LS, Precious E, Crombie IK, Smith WC: Weight loss interventions in young people (18 to 25 year olds): a systematic review. Obes Rev 2010, 11(8):580-592.

24. Eisler RM: The relationship between masculine gender role stress and men's health risk: The validation of a construct. In A New Psychology of Men. Edited by Levant RF, Pollack WS. New York: BasicBooks; 1995:207-225.

25. Neff JA, Prihoda TJ, Hoppe SK: " Machismo," self-esteem, education and high maximum drinking among anglo, black and Mexican-American male drinkers. J Stud Alcohol 1991, 52(5):458-463.

26. O'Neil JM, Good GE, Holmes S: Fifteen years of theory and research on men's gender role conflict: New paradigms for empirical research. In $A$ New Psychology of Men. Edited by Levant RF, Pollack WS. New York: BasicBooks; 1995:164-206.

27. Addis ME, Mahalik JR: Men, masculinity, and the contexts of help seeking. Am Psychol 2003, 58(1):5.
28. Young $K$, White P: Researching sports injury: reconstructing dangerous masculinities. Res Men Masculinities Ser 2000, 13:108-126.

29. White A, De Sousa B, De Visser R, Hogston R, Aage Madsen S, Makara P, Richardson N, Zatonski W: The State of Men's Health in Europe: Extended Report. 2011:1-407.

30. Courtenay WH: Engendering health: a social constructionist examination of men's health beliefs and behaviours. Psychol Men Masculinity 2000, 1(1):4.

31. Oliffe JL, Greaves L: Designing and Conducting Gender, Sex, and Health Research. Thousand Oak, CA: Sage Publishers; 2012.

32. Kreuter MW, Lukwago SN, Bucholtz DC, Clark EM, Sanders-Thompson V: Achieving cultural appropriateness in health promotion programs: targeted and tailored approaches. Health Educ Behav 2003, 30(2):133-146.

33. Courtenay WH: Constructions of masculinity and their influence on men's well-being: a theory of gender and health. Soc Sci Med 2000, 50(10):1385-1401.

34. Barwick H: Young Males: Strengths-Based and Male-Focused Approaches. Wellington: Ministry of Youth Development; 2004.

35. Kreuter MW, Farrell DW, Olevitch LR, Brennan LK: Tailoring Health Messages: Customizing Communication with Computer Technology. New York: Routledge; 2013.

36. Petty RE, Cacioppo JT: A General framework for understanding Attitude change processes. In Attitudes and Persuasion: Classic and Contemporary Approaches. Edited by Petty RE, Cacioppo JT. Dubuque IA: William C. Brown Company; 1981:255-269

37. Petty RT, Cacioppo JT, Strathman AJ, Priester JR: To think or not to think. Exploring two routes to persuasion. In Persuasion Psychological Insights and Perspectives. Edited by Shavitt S, Brock TC. Boston: Allyn and Bacon; 1994:113-147.

38. Young MD, Morgan PJ, Plotnikoff RC, Callister R, Collins CE: Effectiveness of male-only weight loss and weight loss maintenance interventions: a systematic review with meta-analysis. Obes Rev 2012, 13(5):393-408.

39. George ES, Kolt GS, Duncan MJ, Caperchione CM, Mummery WK, Vandelanotte $C$, Taylor $P$, Noakes M: A review of the effectiveness of physical activity interventions for adult males. Sports Med 2012, 42(4):281-300.

40. Okoli CT, Torchalla I, Oliffe JL, Bottorff JL: Men's smoking cessation interventions: a brief review. J Men's Health 2011, 8(2):100-108.

41. Loria CM, Signore C, Arteaga SS: The need for targeted weight-control approaches in young women and men. Am J Prev Med 2010, 38(2):233-235

42. Dour CA, Horacek TM, Schembre SM, Lohse B, Hoerr S, Kattelmann K, White AA, Shoff S, Phillips B, Greene G: Process evaluation of $<\mathrm{i}>$ Project WebHealth $</ \mathrm{i}>$ : a nondieting web-based intervention for obesity prevention in college students. J Nutr Educ Behav 2013, 45(4):288-295.

43. Burns J, Davenport TA, Christensen H, Luscombe GM, Mendoza JA, Bresnan A, Blanchard ME, Hickie IB: Game On: Exploring the Impact of Technologies on Young Men's Mental Health and Wellbeing. Findings from the first Young and Well National Survey. In Melbourne: Young and Well Cooperative Research Centre; 2013:1-76. http://www.youngandwellcrc. org.au/wp-content/uploads/2014/03/Young_and_Well_CRC_Game_On Report.pdf.

44. Harper GW, Riplinger AJ: HIV prevention interventions for adolescents and young adults: what about the needs of gay and bisexual males? AIDS Behav 2013, 17(3):1082-1095.

45. Bingham CR, Barretto Al, Walton MA, Bryant CM, Shope JT, Raghunathan TE: Efficacy of a web-based, tailored, alcohol prevention/intervention program for college students: 3-month follow-up. J Drug Educ 2011 41(4):405-430

46. Arksey H, O'Malley L: Scoping studies: towards a methodological framework. Int J Soc Res Meth 2005, 8(1):19-32

47. Trials use technology to help young adults achieve healthy weights. In http://www.nih.gov/news/health/nov2010/nhlbi-29.htm

48. Country and Lending groups. In http://data.worldbank.org/about/countryand-lending-groups\#Low_income.

49. Welte JW, Barnes GM, Tidwell M-CO, Hoffman JH: The prevalence of problem gambling among US adolescents and young adults: Results from a national survey. J Gamb/ Stud 2008, 24(2):119-133.

50. Levac D, Colquhoun H, O'Brien KK: Scoping studies: advancing the methodology. Implement Sci 2010, 5:69.

51. Bingham TC-H J: Evaluating Locally Developed Homegrown HIV Prevention Interventions. In 2013. Current Controlled Trials. http://clinicaltrials.gov/ct2/ show/NCT01640392. 
52. Ahola R, Pyky R, Jamsa T, Mantysaari M, Koskimaki H, Ikaheimo TM, Huotari ML, Roning J, Heikkinen HI, Korpelainen R: Gamified physical activation of young men-a Multidisciplinary Population-Based Randomized Controlled Trial (MOPO study). BMC Public Health 2013, 13:32.

53. Akbarpour $M$ : The effect of aerobic training on serum adiponectin and leptin levels and inflammatory markers of coronary heart disease in obese men. Biol Sport 2013, 30(1):21-27.

54. Anshel $\mathrm{MH}$ : Effect of chronic aerobic exercise and progressive relaxation on motor performance and affect following acute stress. Behav Med 1996, 21(4):186-196.

55. Asci F: Sex differences in psychological effects of exercise. Int J Psychol 2009, 44(4):313-320.

56. Asci FH: The effects of step dance on physical self-perception of female and male university students. Int J Sport Psychol 2002, 33(4):431-442.

57. Bond V, Bartels MN, Sloan RP, Millis RM, Zion AS, Andrews N, De Meersman RE: Exercise training favourably affects autonomic and blood pressure responses during mental and physical stressors in African-American men. J Hum Hypertens 2009, 23(4):267-273.

58. Bond V, Stephens Q, Adams RG, Vaccaro P, Demeersman R, Williams D, Obisesan TO, Franks BD, Oke LM, Coleman B, Blakely R, Millis RM: Aerobic exercise attenuates an exaggerated exercise blood pressure response in normotensive young adult African-American men. Blood Press 2002 11(4):229-234

59. Calfas KJ, Sallis JF, Nichols JF, Sarkin JA, Johnson MF, Caparosa S, Thompson S, Gehrman CA, Alcaraz JE: Project GRAD: Two-year outcomes of a randomized controlled physical activity intervention among young adults. Am J Prev Med 2000, 18(1):28-37.

60. Cederberg H, Koivisto VM, Jokelainen J, Surcel HM, Keinanen-Kiukaanniemi S, Rajala U: Unacylated ghrelin is associated with changes in insulin sensitivity and lipid profile during an exercise intervention. Clin Endocrinol (Oxf) 2012, 76(1):39-45.

61. Cederberg H, Mikkola I, Jokelainen J, Harkonen P, Ikaheimo T, Laakso M, Keinanen-Kiukaanniemi S: Exercise during military training improves cardiovascular risk factors in young men. Atherosclerosis 2011, 216(2):489-495.

62. Donnelly JE, Hill JO, Jacobsen DJ, Potteiger J, Sullivan DK, Johnson SL, Heelan K, Hise M, Fennessey PV, Sonko B, Sharp T, Jakicic JM, Blair SN, Tran ZV, Mayo M, Gibson C, Washburn RA: Effects of a 16-month randomized controlled exercise trial on body weight and composition in young, overweight men and women: the Midwest Exercise Trial. Arch Int Med 2003, 163:1343-1350.

63. Donnelly JE, Washburn RA, Smith BK, Sullivan DK, Gibson C, Honas JJ, Mayo MS: A randomized, controlled, supervised, exercise trial in young overweight men and women: the Midwest Exercise Trial II (MET2). Contemp Clin Trials 2012, 33:804-810.

64. Ghahramanloo E, Midgley AW, Bentley DJ: The effect of concurrent training on blood lipid profile and anthropometrical characteristics of previously untrained men. J Phys Act Health 2009, 6:760-766.

65. Heydari M, Boutcher YN, Boutcher SH: High-intensity intermittent exercise and cardiovascular and autonomic function. Clin Auton Res 2013, 23(1):57-65

66. Heydari M, Boutcher YN, Boutcher SH: The effects of high-intensity intermittent exercise training on cardiovascular response to mental and physical challenge. Int J Psychophysio/ 2013, 87(2):141-146.

67. Hiruntrakul A, Nanagara R, Emasithi A, Borer KT: Effect of once a week endurance exercise on fitness status in sedentary subjects. J Med Assoc Thai 2010, 93:1070-1074

68. Kirk EP, Washburn RA, Bailey BW, LeCheminant JD, Donnelly JE: Six month of supervised high-intensity low-volume resistance training improves strength independent of changes in muscle mass in young overweight men. J Strength Cond Res 2007, 21:151-156.

69. Marra C, Bottaro M, Oliveira RJ, Novaes JS: Effect of moderate and high intensity aerobic exercise on the body composition of overweight men J Exerc Physiol Online 2005, 8(2):39-45

70. Ozdemir RA, Celik O, Asci FH: Exercise interventions and their effects on physical self-perceptions of male university students. Int J Psychol 2010, 45(3):174-181.

71. Raz I, Israeli A, Rosenblit H, Bar-On H: Influence of moderate exercise on glucose homeostasis and serum testosterone in young men with low HDL-cholesterol level. Diabetes Res 1988, 9:31-35.
72. Raz I, Rosenblit H, Kark JD: Effect of moderate exercise on serum lipids in young men with low high density lipoprotein cholesterol. Arteriosclerosis 1988, 8(3):245-251

73. Roberts CK, Croymans DM, Aziz N, Butch AW, Lee CC: Resistance training increases SHBG in overweight/obese, young men. Metabolism 2013, 62(5):725-733.

74. Rossomanno Cl, Herrick JE, Kirk SM, Kirk EP: A 6-month supervised employer-based minimal exercise program for police officers improves fitness. J Strength Cond Res 2012, 26(9):2338-2344.

75. Sallis JF, Calfas KJ, Nichols JF, Sarkin JA, Johnson MF, Caparosa S, Thompson S, Alcaraz JE: Evaluation of a university course to promote physical activity: Project GRAD. Res Q Exerc Sport 1999, 70(1):1-10.

76. Shaw I, Shaw BS: Relationship between resistance training and lipoprotein profiles in sedentary male smokers. Cardiovasc 2008 19(4):194-197.

77. Spalding TW, Lyon LA, Steel DH, Hatfield BD: Aerobic exercise training and cardiovascular reactivity to psychological stress in sedentary young normotensive men and women. Psychophysiol 2004, 41:552-562.

78. Washburn RA, Donnelly JE, Smith BK, Sullivan DK, Marquis J, Herrmann SD: Resistance training volume, energy balance and weight management: rationale and design of a 9 month trial. Contemp Clin Trials 2012, 33:749-758.

79. Antunes MC, Stall RD, Paiva V, Peres CA, Paul J, Hudes M, Hearst N: Evaluating an AIDS sexual risk reduction program for young adults in public night schools in Sao Paulo, Brazil. AIDS Supp/ 1997 11(1):S121-S127.

80. Armstrong BJ, Kalmuss D, Franks M, Hecker G, Bell D: Creating teachable moments: a clinic-based intervention to improve young men's sexual health. Am J 2010, 4(2):135-144.

81. Borgia P, Marinacci C, Schifano P, Perucci CA: Is peer education the best approach for HIV prevention in schools? Findings from a randomized controlled. J Adolesc Health 2005, 36(6):508-516.

82. Crosby R, DiClemente RJ, Charnigo R, Snow G, Troutman A: A brief, clinicbased, safer sex intervention for heterosexual African American men newly diagnosed with an STD: a randomized controlled trial. Am J Public Health 2009, 99:596-103.

83. Garcia PJ, Holmes KK, Carcamo CP, Garnett GP, Hughes JP, Campos PE, Whittington WLH, Peru PST: Prevention of sexually transmitted infections in urban communities (Peru PREVEN): a multicomponent communityrandomised controlled trial.[Erratum appears in Lancet. 2012 Mar 24;379 (9821):1102]. Lancet 2012, 379(9821):1120-1128.

84. Grant RM, Lama JR, Anderson PL, McMahan V, Liu AY, Vargas L, Goicochea P, Casapía M, Guanira-Carranza JV, Ramirez-Cardich ME, Montoya-Herrera O, Fernández T, Veloso VG, Buchbinder SP, Chariyalertsak S, Schechter M, Bekker LG, Mayer KH, Kallás EG, Amico KR, Mulligan K, Bushman LR, Hance RJ, Ganoza C, Defechereux P, Postle B, Wang F, McConnell JJ, Zheng JH, Lee J, et al: Preexposure chemoprophylaxis for HIV prevention in men who have sex with men. New Eng J Med 2010, 363:2587-2599.

85. Hightow-Weidman LB, Pike E, Fowler B, Matthews DM, Kibe J, McCoy R, Adimora AA: HealthMpowerment.org: feasibility and acceptability of delivering an internet intervention to young Black men who have sex with men. AIDS Care - Psychological and Socio-Medical Aspects of AIDS/HIV 2012, 24(7):910-920.

86. Hosek SG, Siberry G, Bell M, Lally M, Kapogiannis B, Green K, Fernandez MI, Rutledge B, Martinez J, Garofalo R, Wilson CM: The acceptability and feasibility of an HIV preexposure prophylaxis (PrEP) trial with young men who have sex with men. J Acquir Immune Defic Syndr 2013, 62(4):447-456.

87. Kalmuss D, Armstrong B, Franks M, Hecker G, Gonzalez J: Evaluation of a community-based sexual health intervention for young adult Latino and African-American men. J Men's Health 2008, 5(4):318-326.

88. Kegeles SM, Hays RB, Coates TJ: The Mpowerment Project: a community-level HIV prevention intervention for young gay men. Am J Public Health 1996, 86(8, Pt 1):1129-1136.

89. Kegeles SM, Hays RB, Pollack LM, Coates TJ: Mobilizing young gay and bisexual men for HIV prevention: A two-community study. AIDS 1999, 13(13):1753-1762

90. Kennedy SB, Nolen S, Pan ZF, Smith B, Applewhite J, Vanderhoff KJ: Effectiveness of a brief condom promotion program in reducing risky sexual behaviours among African American men. J Eval Clin Pract 2013, 19(2):408-413. 
91. Milhausen RR, Wood J, Sanders SA, Crosby RA, Yarber WL, Graham CA: A novel, self-guided, home-based intervention to promote condom use among young men: a pilot study. J Men's Health 2011 8(4):274-281.

92. Moyer-Gusé E, Nabi RL: Comparing the effects of entertainment and educational television programming on risky sexual behavior. Health Commun 2011, 26:416-426.

93. Sanderson CA: Role of relationship context in influencing college students' responsiveness to HIV prevention videos. Health Psychol 1999, 18(3):295-300.

94. Somerville GG, Diaz S, Davis S, Coleman KD, Taveras S: Adapting the popular opinion leader intervention for latino young migrant men who have sex with men. AIDS Educ Prev 2006, 18(Supp|A):137-148.

95. Tyden T, Bergholm M, Hallen A, Odlind V, Olsson S-E, Sjoden P-O, Strand A Bjorkelund C: Evaluation of an STD-prevention program for Swedish university students. J Am Coll Health 1998, 47(2):70-75

96. Weisse CS, Turbiasz AA, Whitney DJ: Behavioral training and aids risk reduction: Overcoming barriers to condom use. AIDS EduC Prev 1995, 7(1):50-59.

97. Wolitski RJ: Relative efficacy of a multisession sexual risk-reduction intervention for young men released from prisons in 4 states. Am J Public Health 2006, 96(10):1854-1861.

98. Shepherd J, Weare K, Turner G: Peer-led sexual health promotion with young gay and bisexual men-results of The HAPEER Project. Health Educ 1997, 97(6):204-212

99. Ha EJ, Caine-Bish N, Holloman C, Lowry-Gordon K: Evaluation of effectiveness of class-based nutrition intervention on changes in soft drink and milk consumption among young adults. Nutr J 2009, 8:50

100. Ha E-J, Caine-Bish N: Effect of nutrition intervention using a general nutrition course for promoting fruit and vegetable consumption among college students. J Nutr Educ Behav 2009, 41(2):103-109.

101. Ha E-J, Caine-Bish N: Interactive introductory nutrition course focusing on disease prevention increased whole-grain consumption by college students. J Nutr Educ Behav 2011, 43(4):263-267.

102. Sandström B, Marckmann P, Bindslev N: An eight-month controlled study of a low-fat high-fibre diet: effects on blood lipids and blood pressure in healthy young subjects. Eur J Clin Nutr 1992, 46:95-109.

103. Williams DR, Lewis NM: Effectiveness of nutrition counseling in young adult males. Nutr Res 2002, 22(8):911-917.

104. Falk B, Montgomery H: Promoting traffic safety among young male drivers by means of elaboration-based interventions. Transport Res $F$ Traffic Psychol Behav 2009, 12(1):1-11.

105. Lenne MG, Liu CC, Salmon PM, Holden M, Moss S: Minimising risks and distractions for young drivers and their passengers: An evaluation of a novel driver-passenger training program. Transport Res $F$ Traffic Psychol Behav 2011, 14(6):447-455.

106. Rivara FP, Boisvert D, Relyea-Chew A, Gomez T: Last Call: decreasing drunk driving among 21-34-year-old bar patrons. Int J Inj Contr Saf Promot 2012, 19(1):53-61.

107. Taubman-Ben-Ari O, Lotan T: The contribution of a novel intervention to enhance safe driving among young drivers in Israel. Accid Anal Prev 2011, 43(1):352-359.

108. White MJ, Cunningham LC, Titchener K: Young drivers' optimism bias for accident risk and driving skill: Accountability and insight experience manipulations. Accid Anal Prev 2011, 43:1309-1315.

109. Berecz JM: Reduction of cigarette smoking through self-administered aversion conditioning: a new treatment model with implications for public health. Soc Sci Med 1972, 6:57-66.

110. Ergul $\mathrm{S}$, Temel $A B$ : The effects of a nursing smoking cessation intervention on military students in Turkey. Int Nurs Rev 2009, 56(1):102-108.

111. Prokhorov AV, Yost T, Mullin-Jones M, de Moor C, Ford KH, Marani S, Kilfoy BA, Hein JP, Hudmon KS, Emmons KM: "Look At Your Health": outcomes associated with a computer-assisted smoking cessation counseling intervention for community college students. Addict Behav 2008, 33(6):757-771

112. Simmons VN, Brandon TH: Secondary smoking prevention in a university setting: A randomized comparison of an experiential, theory-based intervention and a standard didactic intervention for increasing cessation motivation. Health Psychol 2007, 26(3):268-277.

113. Lee CM, Neighbors C, Kilmer JR, Larimer ME: A brief, web-based personalized feedback selective intervention for college student marijuana use: a randomized clinical trial. Psychol Addict Behav 2010, 24:265-273.

114. Wu Z, Detels R, Zhang J, Li V, Li J: Community-based trial to prevent drug use among youths in Yunnan, China. Am J Public Health 2002, 92:1952-1957

115. Walsh LA, Stock ML: UV photography, masculinity, and college men's sun protection cognitions. J Behav Med 2012, 35(4):431-442.

116. Cambien F, Richard JL, Ducimetiere P, Warnet JM, Kahn J: The Paris Cardiovascular Risk Factor Prevention Trial. Effects of two years of intervention in a population of young men. J Epidemiol Community Health 1981, 35:91-97.

117. Denering LL, Spear SE: Routine use of screening and brief intervention for college students in a university counseling center. J Psychoactive Drugs 2012, 44(4):318-324.

118. Dermen $\mathrm{KH}$, Thomas SN: Randomized controlled trial of brief interventions to reduce college students' drinking and risky sex. Psychol Addict Behav 2011, 25(4):583-594.

119. Gmel G, Gaume J, Bertholet N, Fluckiger J, Daeppen JB: Effectiveness of a brief integrative multiple substance use intervention among young men with and without booster sessions. J Subst Abuse Treat 2013, 44(2):231-240

120. Hebden L, Balestracci K, McGeechan K, Denney-Wilson E, Harris M, Bauman A Allman-Farinelli M: 'TXT2BFiT' a mobile phone-based healthy lifestyle program for preventing unhealthy weight gain in young adults: study protocol for a randomized controlled trial. Trials 2013, 14(75):1-9.

121. Kazemi DM, Dmochowski J, Sun L, Grady K, Nies MA, Walford S: Brief motivational interviewing to reduce alcohol consumption among freshmen: Secondary effects on polydrug use. J Subst Use 2012, 17(5-6):442-455.

122. Shorey RL, Sewell B, O'Brien M: Efficacy of diet and exercise in the reduction of serum cholesterol and triglyceride in free-living adult males. Am J Clin Nutr 1976, 29:512-521.

123. Ulla Diez SM, Fortis AP, Franco SF: Efficacy of a health-promotion intervention for college students. Nurs Res 2012, 61(2):121-132

124. Howat $P$, Robinson S, Binns C, Palmer S: Educational biofeedback driving simulator as a drink-driving prevention strategy. J Alcohol Drug Educ 1991, 37(1):7-14

125. Murphy TJ, Pagano RR, Marlatt GA: Lifestyle modification with heavy alcohol drinkers: Effects of aerobic exercise and meditation. Addict Behav 1986, 11(2):175-186

126. Caudill BD, Luckey B, Crosse SB, Blane HI, Ginexi EM, Campbell B: Alcohol risk-reduction skills training in a national fraternity: A randomized intervention trial with longitudinal intent-to-treat analysis. J Stud Alcohol Drugs 2007, 68(3):399-409.

127. Daeppen JB, Bertholet N, Gaume J, Fortini C, Faouzi M, Gmel G: Efficacy of brief motivational intervention in reducing binge drinking in young men: A randomized controlled trial. Drug Alcohol Depend 2011, 113(1):69-75

128. Gaume J, Gmel G, Faouzi M, Bertholet N, Daeppen JB: Is brief motivational intervention effective in reducing alcohol use among young men voluntarily receiving it? A randomized controlled trial. Alcohol Clin Exp Res 2011, 35(10):1822-1830.

129. Rohsenow DJ, Smith RE, Johnson S: Stress management training as a prevention program for heavy social drinkers: cognitions, affect, drinking, and individual differences. Addict Behav 1985, 10(1):45-54.

130. Baer JS, Kivlahan DR, Blume AW, McKnight P, Marlatt GA: Brief intervention for heavy-drinking college students: 4-year follow-up and natural history. Am J Public Health 2001, 91(8):1310-1316.

131. Barnett NP, Apodaca TR, Magill M, Colby SM, Gwaltney C, Rohsenow DJ, Monti PM: Moderators and mediators of two brief interventions for alcohol in the emergency department. Addiction 2010, 105(3):452-465.

132. Bingham C, Barretto Al, Walton MA, Bryant CM, Shope JT, Raghunathan TE: Efficacy of a Web-based, tailored, alcohol prevention/intervention program for college students: Initial findings. J Am Coll Health 2010, 58(4):349-356.

133. Blow FC, Barry KL, Walton MA, Maio RF, Chermack ST, Bingham C, Ignacio RV, Strecher VJ: The efficacy of two brief intervention strategies among injured, at-risk drinkers in the Emergency Department: impact of tailored messaging and brief advice. J Stud Alcohol 2006, 67(4):568-578.

134. Carey KB, Carey MP, Maisto SA, Henson JM: Brief motivational interventions for heavy college drinkers: A randomized controlled trial. J Consult Clin Psychol 2006, 74(5):943-954. 
135. Davidson D, Swift R, Fitz E: Naltrexone increases the latency to drink alcohol in social drinkers. Alcohol Clin Exp Res 1996, 20:732-739.

136. Dunn ME, Cathy Lau H, Cruz IY: Changes in activation of alcohol expectancies in memory in relation to changes in alcohol use after participation in an expectancy challenge program. Exp Clin Psychopharmacol 2000, 8(4):566-575.

137. Fournier AK, Ehrhart IJ, Glindemann KE, Geller E: Intervening to decrease alcohol abuse at university parties: differential reinforcement of intoxication level. Behav Modif 2004, 28(2):167-181.

138. Hembroff L, Atkin C, Martell D, McCue C, Greenamyer JT: Evaluation results of a 21st birthday card program targeting high-risk drinking. J Am Coll Health 2007, 56(3):325-332.

139. Hester RK, Delaney HD, Campbell W: The College Drinker's Check-Up: outcomes of two randomized clinical trials of a computer-delivered intervention. Psychol Addict Behav 2012, 26(1):1-12.

140. Ichiyama MA, Fairlie AM, Wood MD, Turrisi $R$, Francis DP, Ray AE, Stanger LA: A randomized trial of a parent-based intervention on drinking behaviour among incoming college freshmen. J Stud Alcohol Drugs 2009, 16:67-76.

141. Kazemi D, Dmochowski J, Sun L, Nies M, Walford S: Outcomes of a targeted capacity expansion (TCE) brief motivational intervention for high-risk drinking freshmen: Pilot study comparison at baseline and 6 months. Alcohol Treat Q 2011, 29(3):219-229.

142. Labrie J, Migliuri S, Cail J: A night to remember: a harm-reduction birthday card intervention reduces high-risk drinking during 21st birthday celebrations. J Am Coll Health 2009, 57(6):659-663.

143. Lewis MA, Neighbors C, Lee CM, Oster-Aaland L: 21st birthday celebratory drinking: Evaluation of a personalized normative feedback card intervention. Psychol Addict Behav 2008, 22(2):176-185.

144. Marlatt G, Baer JS, Kivlahan DR, Dimeff LA, Larimer ME, Quigley LA, Somers $J M$, Williams E: Screening and brief intervention for high-risk college student drinkers: Results from a 2-year follow-up assessment. J Consult Clin Psychol 1998, 66(4):604-615.

145. Neighbors C, Lee CM, Atkins DC, Lewis MA, Kaysen D, Mittmann A, Fossos $\mathrm{N}$, Geisner IM, Zheng C, Larimer ME: A randomized controlled trial of event-specific prevention strategies for reducing problematic drinking associated with 21st birthday celebrations. J Consult Clin Psychol 2012, 80(5):850-862

146. Wood MD, Capone C, Laforge R, Erickson DJ, Brand NH: Brief motivational intervention and alcohol expectancy challenge with heavy drinking college students: A randomized factorial study. Addict Behav 2007, 32(11):2509-2528

147. Wood MD, Fairlie AM, Fernandez AC, Borsari B, Capone C, Laforge R, Carmona-Barros R: Brief motivational and parent interventions for college students: A randomized factorial study. J Consult Clin Psychol 2010, 78(3):349-361.

148. Voogt CV, Poelen EAP, Kleinjan M, Lemmers L, Engels R: Targeting young drinkers online: the effectiveness of a web-based brief alcohol intervention in reducing heavy drinking among college students: study protocol of a two-arm parallel group randomized controlled trial. BMC Public Health 2011, 11:1-8

149. Kypri K, McCambridge J, Vater T, Bowe SJ, Saunders JB, Cunningham JA, Horton NJ: Web-based alcohol intervention for Maori university students: double-blind, multi-site randomized controlled trial. Addiction 2013, 108(2):331-338

150. Morgan PJ, Callister R, Collins CE, Plotnikoff RC, Young MD, Berry N, McElduff P, Burrows T, Aguiar E, Saunders KL: The SHED-IT community trial: A randomized controlled trial of internet-and paper-based weight loss programs tailored for overweight and obese men. Ann Behav Med 2013, 45(2):139-152

151. Morgan PJ, Lubans DR, Collins CE, Warren JM, Callister R: 12-month outcomes and process evaluation of the SHED-IT RCT: an internet-based weight loss program targeting men. Obesity 2011, 19(1):142-151.

152. Mullen K, Watson J, Swift J, Black D: Young men, masculinity and alcohol. Drugs Educ Prev Pol 2007, 14(2):151-165.

153. Connell RW, Messerschmidt JW: Hegemonic masculinity rethinking the concept. Gend Soc 2005, 19(6):829-859.

154. Glanz K, Rimer BK, Viswanath K: Health Behaviour and Health Education: Theory, Research, and Practice. San Francisco: John Wiley \& Sons; 2008.

155. Webb TL, Joseph J, Yardley L, Michie S: Using the internet to promote health behaviour change: a systematic review and meta-analysis of the impact of theoretical basis, use of behaviour change techniques, and mode of delivery on efficacy. J Med Internet Res 2010, 12(1):e4.

156. Grol RP, Bosch MC, Hulscher ME, Eccles MP, Wensing M: Planning and studying improvement in patient care: the use of theoretical perspectives. Milbank Q 2007, 85(1):93-138.

157. Evans J, Frank B, Oliffe JL, Gregory D: Health, illness, men and masculinities (HIMM): a theoretical framework for understanding men and their health. J Men's Health 2011, 8(1):7-15.

158. Wenger E: Communities of Practice: Learning, Meaning, and Identity Cambridge: Cambridge university press; 1998.

159. Rhodes RE, Nigg CR: Advancing physical activity theory: a review and future directions. Exerc Sport Sci Rev 2011, 39(3):113-119.

160. Weinstein ND: Misleading tests of health behaviour theories. Ann Behav Med 2007, 33(1):1-10.

161. Fergusson DM, Woodward LJ: Family socioeconomic status at birth and rates of university participation. New Zeal J Educ Stud 2000, 35(1):25-36

162. Griffiths F, Lindenmeyer A, Powell J, Lowe P, Thorogood M: Why are health care interventions delivered over the internet? A systematic review of the published literature. J Med Internet Res 2006, 8(2):e10.

163. Dembroski TM, Lasater TM, Ramirez A: Communicator similarity, fear arousing communications, and compliance with health care recommendations1. J App/ Soc Psychol 1978, 8(3):254-269.

164. Archer RL: Self-disclosure. In In the self in Social Psychology. Edited by Wegner DM, Vallacher RR. New York: Oxford University Press; 1980.

165. Morgan PJ, Collins CE, Plotnikoff RC, McElduff P, Burrows T, Warren JM, Young MD, Berry N, Saunders KL, Aguiar EJ, Callister R: The SHED-IT community trial study protocol: a randomised controlled trial of weight loss programs for overweight and obese men. BMC Public Health 2010, 10:701.

166. Morgan PJ, Collins CE, Plotnikoff RC, Callister R, Burrows T, Fletcher R, Okely $A D$, Young MD, Miller A, Lloyd AB: The 'Healthy Dads, Healthy Kids' community randomized controlled trial: a community-based healthy lifestyle program for fathers and their children. Prev Med 2014, 61:90-99.

167. Resnicow K, Baranowski T, Ahluwalia J, Braithwaite R: Cultural sensitivity in public health: defined and demystified. Ethn Dis 1998, 9(1):10-21.

168. Clarke A, Gatineau M, Grimaud O, Royer-Devaux S, Wyn-Roberts N, Le Bis I, Lewison G: A bibliometric overview of public health research in Europe. Eur J Public Health 2007, 17(Suppl 1):43-49.

169. World Health Organization: Men, ageing and health: Achieving Health Across the Life Span. Geneva: World Health Organization; 2001:1-63.

170. Flick U: An Introduction to Qualitative Research. 5th edition. London: Sage; 2014

171. Brien SE, Lorenzetti DL, Lewis S, Kennedy J, Ghali WA: Overview of a formal scoping review on health system report cards. Implement Sci 2010, 5(2):2

172. Schulz KF, Altman DG, Moher D: CONSORT 2010 statement: updated guidelines for reporting parallel group randomised trials. BMC Med 2010, 8(1):18.

173. Stewart LA, Tierney JF: To IPD or not to IPD? Advantages and disadvantages of systematic reviews using individual patient data. Eval Health Prof 2002, 25(1):76-97.

174. Zwolinsky S, McKenna J, Pringle A, Daly-Smith A, Robertson S, White A: Optimizing lifestyles for men regarded as 'hard-to-reach'through top-flight football/soccer clubs. Health Educ Res 2013, 28(3):405-413.

doi:10.1186/1471-2458-14-957

Cite this article as: Ashton et al:: A scoping review of risk behaviour interventions in young men. BMC Public Health 2014 14:957. 